\title{
Performance Estimation of the Mid-Infrared Camera and Spectrometer Aboard SPICA
}

\author{
Hirokazu Kataza $^{*}+$, Itsuki Sakon ${ }^{\dagger}$, Takehiko Wada ${ }^{\star}$, Yuki Sarugaku ${ }^{\star}$, Naofumi Fujishiro ${ }^{\S}$, \\ Yuji Ikeda", Shinji Mitanill, Youichi Ohyama** and Naoto Kobayashi ${ }^{\dagger \dagger}$ \\ *Institute of Space and Astronautical Science \\ Japan Aerospace Exploration Agency, 3-1-1 Yoshinodai \\ Sagamihara, Kanagawa 229-8510, Japan \\ $\dagger$ Department of Astronomy, Graduate School of Science \\ University of Tokyo, 7-3-1 Hongo \\ Bunkyo-ku, Tokyo 113-0033, Japan \\ HInstitute of Space and Astronautical Science \\ Japan Aerospace Exploration Agency, 3-1-1 Yoshinodai \\ Sagamihara, Kanagawa 229-8510, Japan

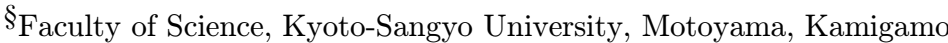 \\ Kita-ku, Kyoto 603-8555, Japan \\ IPhotocoding, 460-102 Iwakura-Nakamachi \\ Sakyo-ku, Kyoto 606-0025, Japan \\ ${ }{ }_{\text {Tsukuba Space Center, Japan Aerospace Exploration Agency, 2-1-1 Sengen }}$ \\ Tsukuba-shi, Ibaraki 305-8505, Japan \\ **Academia Sinica, Institute of Astronomy and Astrophysics \\ No. 1, Sec. 4, Roosevelt Rd, Taipei 10617, Taiwan, ROC \\ †Institute of Astronomy, School of Science \\ University of Tokyo, 2-21-1 Osawa \\ Mitaka, Tokyo 181-0015, Japan \\ +kataza@ir.isas.jaxa.jp
}

Received 2014 August 27; Revised 2014 December 10; Accepted 2014 December 18; Published 2015 March 19

The Space Infrared Telescope for Cosmology and Astrophysics (SPICA) is an astronomical mission optimized for mid- and far-infrared astronomy, envisioned for launch in the 2020s. The Mid-infrared Camera and Spectrometer (MCS) is a model instrument that covers the 5-38 $\mu \mathrm{m}$ wavelength range and enables imaging and spectroscopic observations via four modules named WFC-S, WFC-L, HRS, and MRS. Both of the wide field camera (WFC) modules have a 5-arcmin square field of view (FOV) but cover different wavelength ranges; WFC for the short wavelength region (WFC-S) covers 5 to $24 \mu \mathrm{m}$, whereas WFC for the long wavelength region (WFC-L) covers 18 to $38 \mu \mathrm{m}$. The High Resolution Spectrometer (HRS) covers the 12-18 $\mu \mathrm{m}$ range with a resolving power of 22,000-30,000, and the Mid Resolution Spectrometer (MRS) performs integral filed units spectroscopy with a $12^{\prime \prime}$ by $8^{\prime \prime}$ FOV. MRS simultaneously covers the $12-38 \mu \mathrm{m}$ range with a moderate resolving power of 720-2000. Here, we report sensitivity estimates from a detailed modeling process involving the instrument itself, the telescope, environmental conditions, and the system error budgets. We show that the WFC-S and HRS modules require an adaptive system to correct for telescope pointing error. In particular, band pass filters (BPFs) longer than $26 \mu \mathrm{m}$ should be developed.

Keywords: Mid-infrared, instrument on board, SPICA. 


\section{Introduction}

The fundamental themes of modern astronomy are galactic evolution, the metal enrichment history of the inter-stellar medium (ISM) which is linked to nucleosynthesis, and the formation of stars and planetary systems. These themes are investigated in the mid- to far-infrared wavelength region because gas and dust exhibits rich lines and band features in this region. A large cooled orbiting telescope will enlarge the sensitivity in the mid- to far-infrared region. Space Infrared Telescope for Cosmology and Astrophysics (SPICA) (Nakagawa et al., 2012) is a mission optimized for observation in these wavelength regions and is envisioned for launch in the 2020s. SPICA will investigate three major scientific goals: "the drama of galaxy formation (Resolution of Birth and Evolution of Galaxies)," "the recipe for planetary systems (Thorough Understanding of Planetary System Formation)," and "the Life Cycle of Dust in the Universe." It has a 3 m class telescope cooled to very low temperature (approximately $6 \mathrm{~K}$ ) so that its background thermal emission is negligibly small compared to the natural background from zodiacal emissions. The SPICA instrument suite will consist of two science instruments. The Midinfrared Camera and Spectrometer (MCS) will provide imaging and spectroscopic observing capabilities in the mid-infrared wavelength region (5$38 \mu \mathrm{m})$, whereas the SPICA FAR-infrared Instrument (SAFARI) (Roelfsema et al., 2012) will cover the 35-210 $\mu \mathrm{m}$ wavelength region with a $2 \times 2$ arcmin field of view (FOV). The SAFARI instrument will provide imaging and imaging-Fourier spectroscopic capabilities.

The MCS instrument, with a total wavelength coverage of 5-38 $\mu \mathrm{m}$, comprises four modules with imaging and spectroscopic observing capabilities, namely WFC-S, WFC-L, HRS, and MRS. Both the wide field cameras (WFCs) have a 5-arcmin square FOV, whereas the WFC module for the short wavelength region (WFC-S) covers the $5-24 \mu \mathrm{m}$ region, and the WFC module for the long wavelength region (WFC-L) covers the $18-38 \mu \mathrm{m}$ region. The High-Resolution Spectrometer (HRS) covers the 12$18 \mu \mathrm{m}$ region with a resolving power of 22,000 30,000. The Mid-Resolution Spectrometer (MRS) performs integral filed-units spectroscopy with a $12^{\prime \prime}$ by $8^{\prime \prime}$ FOV. This module covers a wide range (12$38 \mu \mathrm{m})$ with moderate resolving power (720-2000).

MCS is designed to achieve SPICA's abovementioned goals. For instance, by exploiting the wide field imaging capability and high mapping speed of WFC, we can unveil the birth and evolution of galaxies in the early universe. Moreover, the medium resolution spectrographic capability of the MRS module will provide a wide variety of spectral features, from which we can investigate the life cycle of matter in its various physical phases. Meanwhile, the high-resolution spectroscopic capability of the HRS module will reveal the formation mechanism of gaseous giant planets and the initial conditions of terrestrial planet formation.

MCS is a model instrument designed for investigating the performance of the SPICA mission in the mid-infrared region, understanding the required system resources (including developmental resources) of SPICA, and mitigating technical risks. The MCS performances are determined by the telescope parameters, optical design of the instrument, detector performances, performance degradation caused by optical defects, and the pointing stability limits. The system design should consider the scientific merit, estimate the performances of the instrument, and assess the total costs. In this paper, we estimate the sensitivity of MCS based on its design and the estimated errors in the system. Using the values obtained by state-of-the-art technologies, we estimate the errors that can be realized within our expected cost limits. Then, we discuss key items to improve the MCS performances.

\section{Overview of the MCS Instrument}

MCS occupies two areas in SPICA's focal plane (Fig. 1), one covering short wavelengths and the other covering longer wavelengths. The image on the short wavelength part is relayed and re-focused by a fore-optics module installed with a filter/shutter wheel and an optional tip-tilt mirror (TTM). The output image of the fore-optics is divided into two fields, one relayed to WFC-S and the other to HRS. The image on the long wavelength part is relayed and re-focused by another fore-optics module with a filter/shutter wheel, and subsequently divided into fields sent to WFC-L and MRS. Each WFC-S and WFC-L has an individual 5 arcmin square FOV. The edge of the FOV is equipped with short slits, allowing optional grism spectroscopy. HRS and MRS occupy individual FOVs near those of WFC-S and WFC-L, respectively. WFC-S and HRS are installed with a $2 \mathrm{k} \times 2 \mathrm{k}$ Si:As detector, whereas WFC-L has a $1 \mathrm{k} \times$ $1 \mathrm{k}$ Si:Sb detector. MRS is installed with both the 


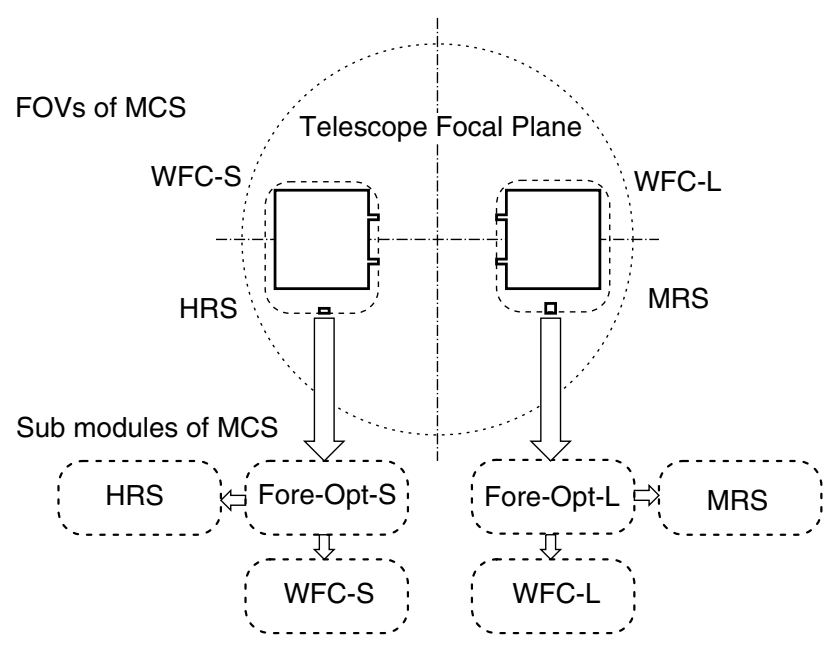

Fig. 1. Overall structure of MCS. The upper and lower parts show the FOV of MCS (not to scale) and the optical modules of MCS, respectively.

detectors. A total of six detectors are adopted in all the modules.

Figure 2 shows the spectroscopic resolving power at different wavelength coverage of MCS. WFC-S and WFC-L have a wide-field imaging capability. The imaging bandwidths of WFC-S and WFC-L are roughly selected as $R(=\lambda / \Delta \lambda)=5$ and 10 , respectively. Optional low resolution $(R=50)$ spectroscopy would be enabled by installing a grism

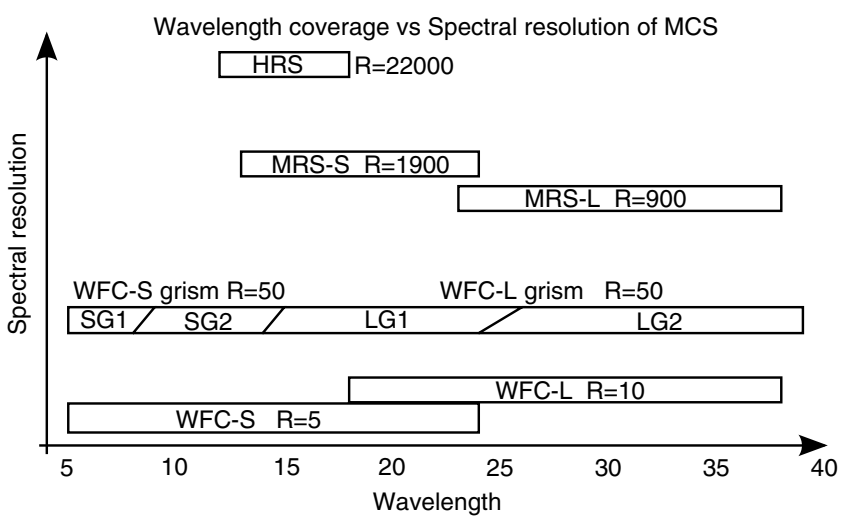

Fig. 2. Wavelength coverage vs. resolving power of MCS.

in the filter wheels. Such an optional mode, which is not accommodated in our present optical design, would achieve wide-FOV objective spectroscopy and short-slit spectroscopy. MRS simultaneously covers a wide wavelength range $(12-38 \mu \mathrm{m})$ because of a dichroic filter placed between its two channels (MRS-S and MRS-L). The integral field unit of each channel is an image slicer sharing a common $12^{\prime \prime} \times$ 8" FOV (Sakon et al., 2013a, 2014). HRS achieves its high resolving power by an immersion grating and optical lens design, which confines its observational capacity within a limited volume.

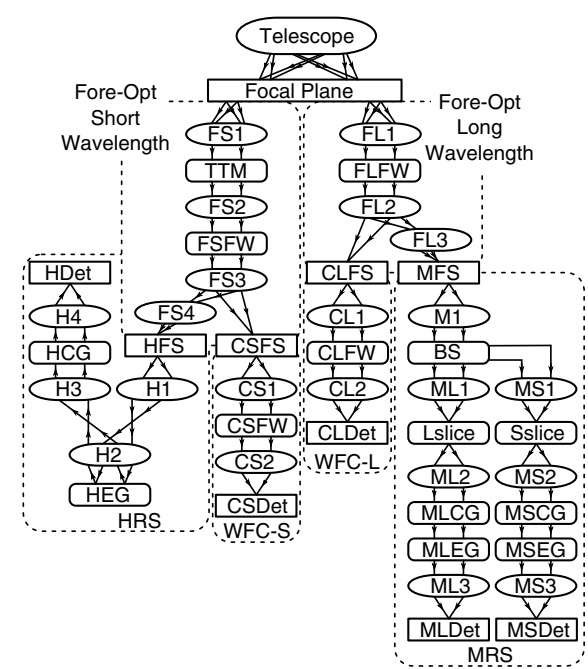

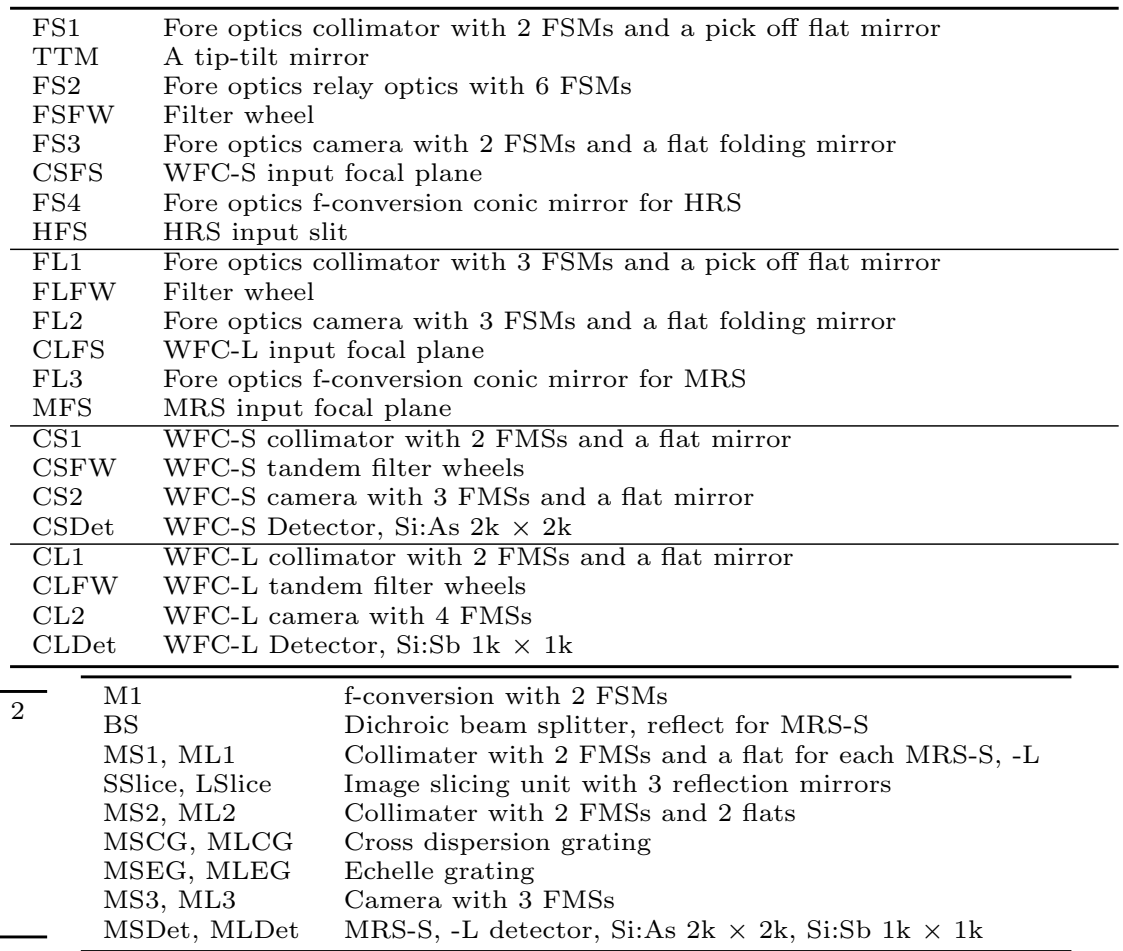

Fig. 3. Optics of the MCS instrument. 
Figure 3 is an optical diagram of MCS. All the optics (the fore-optics, WFC-S/L, and MRS-S/L), excluding the grisms in WFC, employ reflective mirrors. Reflective mirror optics introduce no chromatic aberration and ensure high system transmission (reflectance) throughout the infrared wavelength region. Our free surface mirror (FSM) optics are designed based on the theory by Araki (2005) (Fujishiro et al., 2012).

\section{Model of the System}

\subsection{The SPICA telescope}

The optical parameters (Castel et al., 2012) and transmission characteristics of the SPICA telescope are presented in Tables 1 and 2, respectively. Transmission is defined at intermediate values between clean and dusty (5000 ppm) data collected by the Herschel Space Observatory (HSO). Transmission estimates are linear interpolations between defined points at different wavelengths.

\section{2. $W F C$}

The design parameters of WFC-S/L are listed in Table 3. WFC-S contains 12 mirrors; one telescope tertiary mirror in the fore-optics, two folding mirrors between the fore-optics and the camera, and six mirrors in the camera. WFC-L contains seven mirrors in the fore optics, two mirrors between the fore optics and the camera, and six mirrors in the camera. All the optics (excluding the filters and grisms)

Table 1. The SPICA telescope parameters.

\begin{tabular}{lc}
\hline Entrance pupil diameter $[\mathrm{mm}]$ & 3000 \\
Telescope focal length $[\mathrm{mm}]$ & 16,200 \\
Central obscuration diameter $[\mathrm{mm}]$ & 600 \\
Total obscuration fraction & 0.125 \\
\hline
\end{tabular}

Table 2. SPICA telescope transmission [\%].

\begin{tabular}{cc}
\hline Wavelength $[\mu \mathrm{m}]$ & Transmission \\
\hline 3.5 & 90.0 \\
5 & 92.5 \\
15 & 96.0 \\
30 & 98.0 \\
110 & 99.2 \\
210 & 99.4 \\
\hline
\end{tabular}

are reflective optics with a gold reflection coating. The reflectance of gold is assumed to be constant over the entire wavelength coverage of MCS (Bennett \& Ashley, 1965).

Our design adopts 10-position tandem filter wheels, yielding 20 positions in total. Two positions should be reserved for clear apertures and another position reserved as a blind mask. Thus, 17 filters and grisms are allocated to both of the WFC-S and WFC-L modules. Ten filters and two grisms beginning with $\mathrm{S}$ or $\mathrm{U}$ in Tables 4 and 5 are candidates for WFC-S, whereas those beginning with $\mathrm{L}$ are candidates for WFC-L.

The filter and grism candidates were selected from the scientific requirements as follows (Sakon et al., 2013b). Extragalactic science requires both constant spectral resolving power and continuous spectral coverage to estimate the redshifts from polycyclic aromatic hydrocarbon $(\mathrm{PAH})$ and silicate features. The resolving power $R$ of WFC-S and WFC-R was selected to be 5 and 10, respectively, based on the number of available filter positions. The interstellar medium and planet formation sciences require that the filter set discriminates the emission and absorption features of numerous dust species such as amorphous silicate, crystalline silicate, and astronomical PAHs. Some extragalactic studies further rely on low-resolution spectroscopy of sparsely distributed point sources and high speed multi-object surveying. For this purpose, we installed a selectable grism as an optional component in the camera.

Thin-film multi-layer interference band pass filters (BPFs) covering mid-infrared wavelength regions have been reported. Hawkins \& Sherwood (2008) reported the transmissions of MIRI/JWST filters (Wright et al., 2004). Longer wavelength BPFs require a thicker layer, which is difficult to fabricate given the limited availability of the materials. Very few filters have been fabricated for bandwidths longer than $27 \mu \mathrm{m}$ (Seeley et al., 1981). On the basis of the above-mentioned reports, we assumed BPF transmissions of $80 \%, 70 \%$, and $30 \%$ in the wavelength regions $\lambda_{c}<17 \mu \mathrm{m}, 17 \mu \mathrm{m} \leq \lambda_{c}<26 \mu \mathrm{m}$ and $26 \mu \mathrm{m} \leq \lambda_{c}$, respectively. Here, $\lambda_{c}$ denotes the wavelength at the center of a filter band.

\section{3. $M R S$}

The design parameters of the MRS-S and -L modules are listed in Tables 6 and 7, respectively. In addition to the dichroic beam splitter in the 
Table 3. Optical parameters of WFC.

\begin{tabular}{|c|c|c|}
\hline & WFC-S & WFC-L \\
\hline Mirror reflectance & 0.994 & 0.994 \\
\hline Pixel FoV $[\operatorname{arcsec} / \text { pix }]^{\mathrm{a}}$ & 0.1465 & 0.4125 \\
\hline Nyquist sampling wavelength $[\mu \mathrm{m}]^{\mathrm{a}}$ & 4.26 & 12.00 \\
\hline Effective F-number at the detector & 11.73 & 3.0 \\
\hline Detector full FoV $[\operatorname{arcsec}]^{\mathrm{a}}$ & $300.1 \times 300.1$ & $422.4 \times 422.4$ \\
\hline FOV defined by field stop [arcsec] & $300 \times 293$ & $300 \times 293$ \\
\hline Number of mirrors & 20 & 15 \\
\hline Transmittance excluding filters ${ }^{\mathrm{a}}$ & 0.887 & 0.914 \\
\hline
\end{tabular}

${ }^{\text {a }}$ These values are derived from other values.

Table 4. WFC filter candidates (all wavelengths are in $\mu \mathrm{m}$ ).

\begin{tabular}{lccccc}
\hline Name & Center wavelength & Shortest wavelength & Longest wavelength & Bandwidth & Transmission \\
\hline S4.4 & 4.388 & 3.949 & 4.827 & 0.878 & 0.8 \\
S5.4 & 5.363 & 4.827 & 5.899 & 1.073 & 0.8 \\
S6.6 & 6.555 & 5.899 & 7.210 & 1.311 & 0.8 \\
S8.0 & 8.011 & 7.210 & 8.812 & 1.602 & 0.8 \\
S9.8 & 9.791 & 8.812 & 10.77 & 1.958 & 0.8 \\
S12.0 & 11.97 & 10.77 & 13.16 & 2.393 & 0.8 \\
S14.6 & 14.63 & 13.16 & 16.09 & 2.925 & 0.8 \\
S17.9 & 17.88 & 16.09 & 19.66 & 3.575 & 0.7 \\
S21.8 & 21.85 & 19.66 & 24.03 & 4.370 & 0.7 \\
U11.2 & 11.20 & 11.00 & 11.40 & 0.400 & 0.8 \\
L18.9 & 18.92 & 18.0 & 19.9 & 1.89 & 0.7 \\
L20.8 & 20.81 & 19.8 & 21.9 & 2.08 & 0.7 \\
L22.9 & 22.89 & 21.8 & 24.0 & 2.29 & 0.7 \\
L25.2 & 25.18 & 23.9 & 2.4 & 2.52 & 0.7 \\
L27.7 & 27.70 & 26.3 & 29.1 & 2.77 & 0.3 \\
L30.5 & 30.47 & 29.0 & 32.0 & 3.05 & 0.3 \\
L33.5 & 33.52 & 31.8 & 35.2 & 3.35 & 0.3 \\
L36.9 & 36.87 & 35.0 & 38.7 & 3.69 & 0.3 \\
L27W & 27.00 & 24.0 & 30.0 & 6.0 & 0.3 \\
L34W & 33.75 & 30.0 & 37.5 & 7.5 & 0.3 \\
\hline
\end{tabular}

aThis is an optional filter outside of the main wavelength coverage of WFC.

Table 5. Candidates of WFC grisms (all wavelength are in $\mu \mathrm{m}$ ).

\begin{tabular}{lccccccc}
\hline Name & $\begin{array}{c}\text { Shortest } \\
\text { wavelength }\end{array}$ & $\begin{array}{c}\text { Longest } \\
\text { wavelength }\end{array}$ & $\begin{array}{c}\text { Resolving } \\
\text { power }\end{array}$ & Material & $\begin{array}{c}\text { Pitch } \\
{[\text { lines/mm }]}\end{array}$ & $\begin{array}{c}\text { Prism } \\
\left.\text { angle [ }{ }^{\circ}\right]\end{array}$ & Transmission \\
\hline SG1 & 5 & 9 & 50 & CdTe & 0.320 & 0.7 & NA \\
SG2 & 8 & 15 & 50 & CdTe & 0.320 & 1.0 & NA \\
LG3 & 14 & 26 & 50 & KRS5 & 0.320 & 2.5 & NA \\
LG4 & 24 & 39 & 50 & KRS5 & 0.320 & 4.4 & NA \\
\hline
\end{tabular}

fore-optics, each module is installed with 24 mirrors, two gratings (a cross disperser and an echelle grating), and a BPF. All mirrors are assumed to have the same reflectance with that assumed in WFC.
The throughput of the beam splitter combined with the order-sorting BPF is assumed based on the same assumption for WFC. The wavelength $\lambda_{\text {slit }}$ defines the wavelength at which the diffraction limit scale 
Table 6. MRS optical parameters.

\begin{tabular}{|c|c|c|}
\hline & MRS-S & MRS-L \\
\hline Pixel scale $[\operatorname{arcsec} / \mathrm{pix}]$ & 0.40 & 0.48 \\
\hline Slit width $(w)[\operatorname{arcsec}]$ & 1.60 & 2.67 \\
\hline Slit length $[\operatorname{arcsec}]$ & 12.0 & 12.0 \\
\hline Number of slits in IFU & 5 & 3 \\
\hline Shortest wavelength $[\mu \mathrm{m}]$ & 12.2 & 23.0 \\
\hline Longest wavelength $[\mu \mathrm{m}]$ & 23.5 & 37.8 \\
\hline Wavelength corresponding to the slit width $\left(\lambda_{\text {slit }} / D=w\right)^{\text {a }}$ & 23.27 & 38.83 \\
\hline Number of mirrors & 24 & 24 \\
\hline Dichroic beam splitter + filter throughput & 0.7 & 0.3 \\
\hline Transmittance excluding gratings, slit and filters ${ }^{\mathrm{a}}$ & 0.866 & 0.866 \\
\hline Adjustment factor of total grating efficiency from scalar approx. ${ }^{b}$ & 0.5 & 0.5 \\
\hline
\end{tabular}

aThese values are derived from other values.

${ }^{\mathrm{b}}$ See main text for details.

Table 7. MRS grating parameters.

\begin{tabular}{lccccr}
\hline & \multicolumn{2}{c}{ MRS-S } & & \multicolumn{2}{c}{ MRS-L } \\
\cline { 2 - 3 } \cline { 5 - 6 } & Cross & Main & & Cross & Main \\
\hline Groove spacing $d[\mu \mathrm{m}]$ & 50.0 & 110.0 & & 75.0 & 380.0 \\
Incident angle $\theta_{\text {in }}\left[^{\circ}\right]$ & 10.5 & 11.1 & & 11.6 & 11.9 \\
Blazed output angle $\left[^{\circ}\right]$ & 10.5 & 50.0 & & 11.6 & 47.5 \\
Blaze angle $\beta\left[^{\circ}\right]^{\mathrm{a}}$ & 10.5 & 30.6 & & 11.6 & 29.7 \\
Order & 1 & $5-8$ & & 1 & $10-15$ \\
\hline
\end{tabular}

aThese values are derived from other values.

equals the slit width $\left(w=\lambda_{\text {slit }} / D\right)$. This diffraction limit scale is smaller than the Airy disk first null radius $(1.22 \lambda / D)$. To avoid stray light introduced by the diffraction effect of the slit, $\lambda_{\text {slit }}$ should approximate the longest wavelength covered by the module.

Using the scalar approximation, the dispersion of the output light of a grating at far field is given by (Schroeder, 2000)

$$
\begin{gathered}
I\left(\theta_{\text {out }}\right)=\left(\frac{\sin (u b)}{u}\right)^{2} \cdot\left(\frac{\sin (N d v)}{\sin (d v)}\right)^{2}, \\
u \equiv \pi \frac{\sin \left(\theta_{\text {out }}-\beta\right)+\sin \left(\theta_{\text {in }}-\beta\right)}{\lambda \cos \beta}, \text { and } \\
v \equiv \pi \frac{\sin \theta_{\text {out }}+\sin \theta_{\text {in }}}{\lambda} .
\end{gathered}
$$

Here, $\theta_{\text {in }}$ and $\theta_{\text {out }}$ are the incident and output angles, respectively, $N$ is the number of grooves in a beam, $\beta$ is the blaze angle, $d$ is the groove spacing, and $b$ is the effective groove spacing obtained by subtracting the non-available area from $d$. The first and second terms of Eq. (1) are called the blaze function (BF) and the interference function (IF), respectively.

Let $\theta_{m}$ be the output angle satisfying the $m$ th order interference condition. We assume that IF is non-zero only within a narrow area around $\theta_{m}$, and that $\mathrm{BF}$ is approximately constant in this area. Then, the integral of IF around $\theta_{m}$ is approximately the same for all possible orders, and the grating efficiency is computed as

$$
T_{m}=\left(\frac{\sin \left(u_{m} b\right)}{u_{m}}\right)^{2} / \sum_{k}\left(\frac{\sin \left(u_{k} b\right)}{u_{k}}\right)^{2},
$$

where

$$
u_{m}=\pi \frac{\sin \left(\theta_{m}-\beta\right)+\sin \left(\theta_{\text {in }}-\beta\right)}{\lambda \cos \beta},
$$

and

$$
m \lambda=d\left(\sin \left(\theta_{\text {in }}\right)+\sin \left(\theta_{m}\right)\right) .
$$

The efficiency of the MRS-S gratings was estimated in two ways. First, we applied the abovementioned scalar approximation. We calculated the efficiencies of the cross disperser and the echelle grating by Eq. (1) and normalized them by the sum of contributions from all possible orders. Second, we conducted an electromagnetic wave analysis. In this case, we calculated the efficiencies of the transverse magnetic (TM) mode for the cross grating and transverse electric (TE) mode for the echelle grating and computed the products of the TM (TE) mode efficiencies and the TE (TM) mode efficiencies. The mean values of these two modes were then accepted as the total efficiencies of the gratings. Because these values are smaller than those calculated from 

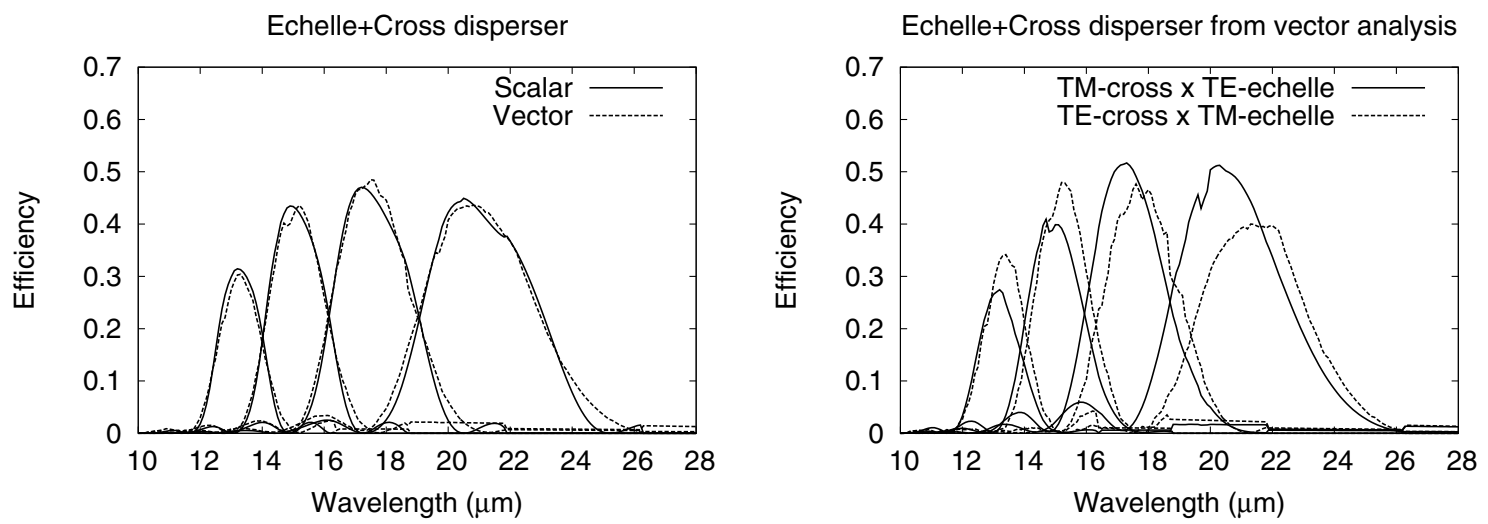

Fig. 4. Efficiency of cross and echelle gratings in MRS-S. The efficiencies in the left panel have been adjusted by the factor given in Table 7. The dashed line is the mean efficiency of both the modes. The right panel shows the efficiencies of each mode. The continuous lines plot the TM mode products for the cross disperser and the TE mode products for the echelle grating. The dashed lines are the products of TE-cross and TM-echelle.

the scalar wave approximation, we adopted the adjustment factor listed in Table 7 . Figure 4 plots the efficiencies calculated by the scalar wave approximation multiplied by the adjustment factor along with the efficiencies obtained by vector analysis. The adjustment factor is selected to ensure the same mean efficiency for both the approximations. According to Fig. 4, the wavelength dependences of the efficiencies obtained by the adjusted scalar approximation and vector electromagnetic analysis closely agree (see Sec. 5 for further discussion). The same adjustment factor was applied to MRS-L.

Figure 5 shows the total efficiencies of the cross and echelle gratings in the MRS-S and -L modules obtained by the scalar approximation with the adjustment factor. The resolving power depends on

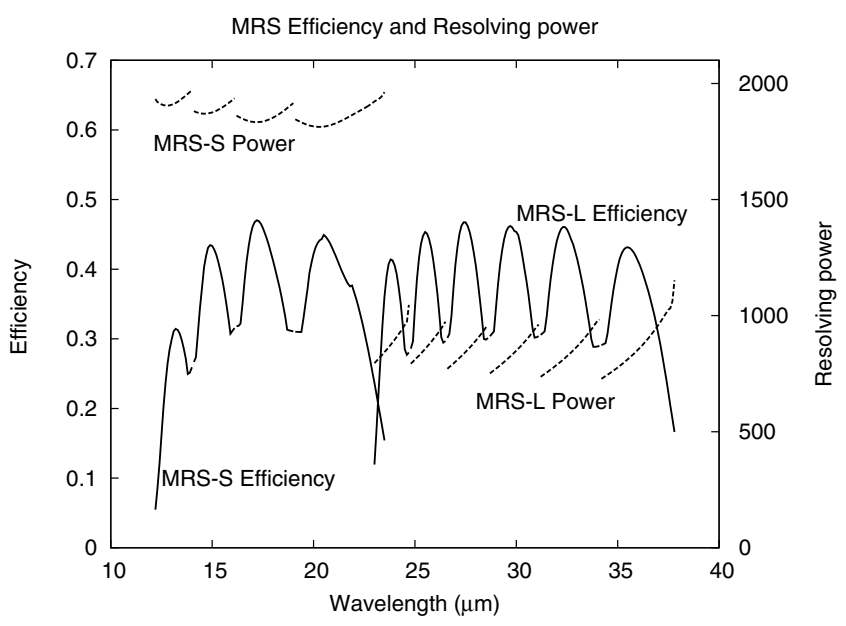

Fig. 5. Total efficiencies (left vertical axis) and resolving power (right vertical axis) of cross and echelle gratings of MRS$\mathrm{S} / \mathrm{L}$. The resolving power is the square root of the sum of the squares of slit width and $\lambda / D$. the slit width and the grating resolution. The former dependency arises from the uncertainty of the center position of a point source within a slit, whereas the latter is governed by $\lambda / D$ and the dispersion of the grating at wavelength $\lambda$. In Fig. 5, the resolving power is the square root of the sum of the squares of the slit width and $\lambda / D$. At some wavelength, signals of the same wavelength are detected in two neighboring orders. In this case, if a high signal-to-noise $(\mathrm{S} / \mathrm{N})$ ratio is expected, the two signals should be summed. In other words, if the efficiencies of an order and its neighboring order are $e 1$ and $e 2$, respectively, at some wavelength, effective efficiency of summed data is calculated as $(e 1+e 2) / \sqrt{2}$ assuming that the same noise is present in both the orders. In the case that this efficiency is higher than $e 1$ or $e 2$ alone, we use this effective efficiency to evaluate sensitivities.

\subsection{HRS}

The design parameters of the HRS module are listed in Tables 8 and 9. In contrast to MRS, HRS combines lens optics with an immersion grating to achieve very high wavelength resolving power within a conservative optical volume. We measured the transparency of an anti-reflection coated $\mathrm{KBr}$ test piece (Fig. 6) and assume the parameters listed in Table 8 for the surface reflection loss of each lens.

Similar to the MRS modules, we estimated the wavelength dependence of the HRS grating efficiency by the scalar approximation. The resolving power is the square root of the sum of the squares of the slit width and $\lambda / D$. The estimated efficiency of the gratings was multiplied by the adjustment 
Table 8. Optical parameters of HRS.

\begin{tabular}{|c|c|}
\hline Pixel scale $[\operatorname{arcsec} / \mathrm{pix}]$ & 0.48 \\
\hline Slit width $(w)[\operatorname{arcsec}]$ & 1.2 \\
\hline Slit length $[\operatorname{arcsec}]$ & 6.0 \\
\hline Shortest wavelength & 11.94 \\
\hline Longest wavelength & 18.07 \\
\hline Wavelength corresponding to the slit width $\left(\lambda_{\text {slit }} / D=w\right)^{\text {a }}$ & 17.45 \\
\hline Number of mirrors & 20 \\
\hline Transmission of mirrors ${ }^{a, b}$ & 0.887 \\
\hline Number of lens surfaces & 32 \\
\hline Transmission of a lens surface & 0.97 \\
\hline Transmission of all lenses ${ }^{\mathrm{a}}$ & 0.377 \\
\hline BPF efficiency & 0.8 \\
\hline Immersion grating transmission $^{\mathrm{c}}$ & 0.8 \\
\hline Adjustment factor of total grating efficiency from scalar appro & 0.5 \\
\hline
\end{tabular}

aThese values are derived from other values.

${ }^{\mathrm{b}}$ The transmission arises from the fore-optics part and folding mirrors.

'Transparent surface loss and mean material transmission within the grating are included. Grating diffraction efficiency is excluded.

Table 9. Grating parameters of HRS.

\begin{tabular}{lcc}
\hline & Cross & Main \\
\hline Groove spacing $d[\mu \mathrm{m}]$ & 25.0 & 281.2 \\
Incident angle $\theta_{\text {in }}\left[^{\circ}\right]$ & 20.0 & 75.0 \\
Blazed output angle $\left[^{\circ}\right]$ & 19.9 & 75.0 \\
Blaze angle $\beta\left[^{\circ}\right]^{\mathrm{a}}$ & 19.9 & 75.0 \\
Order & 1 & $80-121$ \\
Blaze wavelength $\lambda_{B}[\mu \mathrm{m}]^{\mathrm{a}}$ & 17.060 & \\
Groove effective width $b[\mu \mathrm{m}]^{\mathrm{a}}$ & 22.097 & 18.837 \\
Immersion material $^{-}$ & - & CdZnTe \\
\hline
\end{tabular}

aThese values are derived from other values.

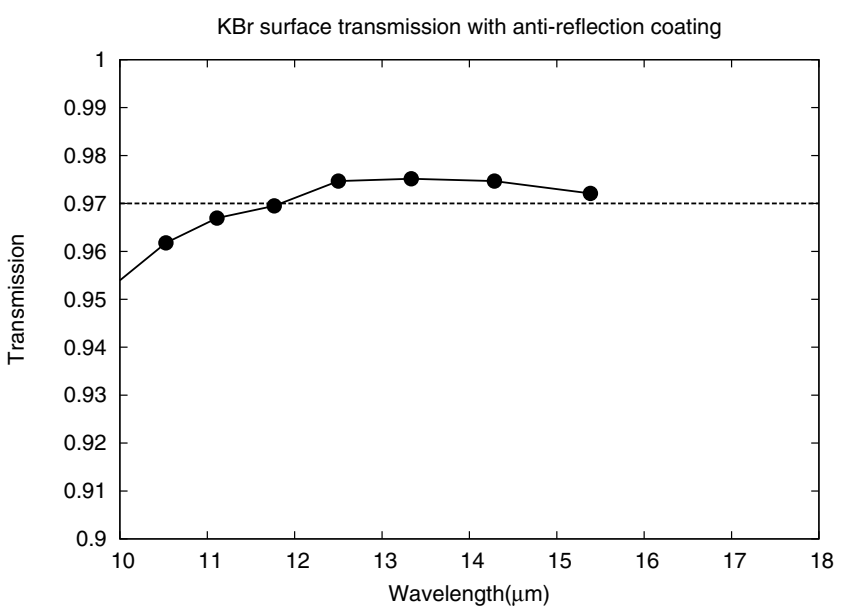

Fig. 6. Transmission at $\mathrm{KBr}$ surface with anti-reflection coating. The dashed line is used to estimate the HRS performance in this paper. factor given in Table 8, and is plotted as a function of wavelength in Fig. 7. Note that the transmission of the immersion grating is excluded in this plot. To calculate the overall sensitivity, the grating efficiency must be multiplied by the transmission.

\subsection{Detector performances}

The parameters and performances of the detectors are listed in Table 10. We provide the state-of-theart performance (SOAP) and goal performance (GOAL), but hereafter use the SOAP values unless otherwise stated.

\subsection{Cosmic ray environment and exposure time}

The longest exposure time is defined such that the mean number of pixels intercepted by cosmic ray remnants during an exposure does not exceed the critical value. The assumed values and calculated maximum exposure time are shown in Table 11 . The cosmic ray hit event rate at L2 was extracted from Swinyard et al. (2004).

\subsection{Detector operation scheme}

The detector readout operation scheme is shown in Fig. 8. In this scheme, the array maintains the same frame readout operation at a fixed frame sampling rate. The first frame (the reset frame) is followed by $N / 2$ frames for measuring the voltage at the start of the exposure. The final $N / 2$ frames are used to 


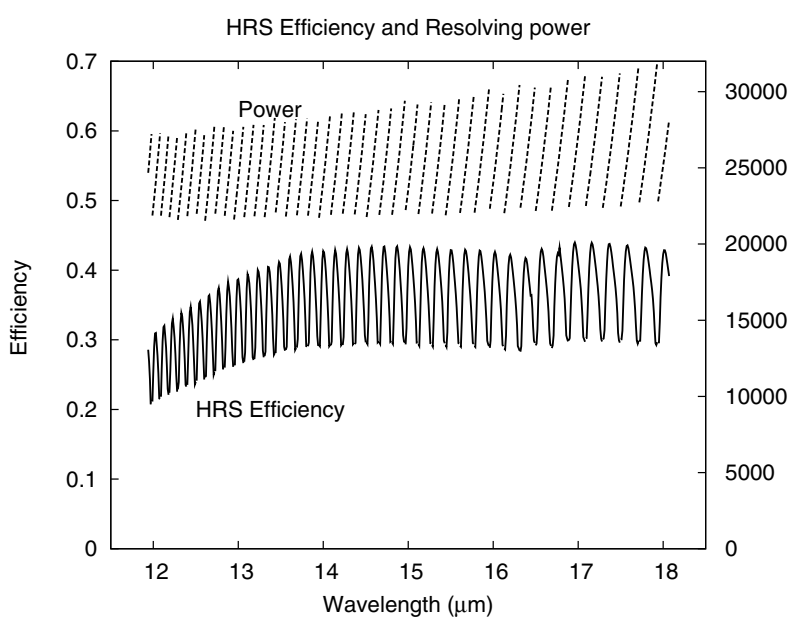

Fig. 7. Total efficiencies (left vertical axis) and resolving power (right vertical axis) of the cross and echelle gratings in HRS. The resolving power is the square root of the sum of the squares of slit width and $\lambda / D$. measure the end point of the exposure. This sampling strategy is called Fowler- $N$ sampling. We set the number of Fowler samplings to 16, ensuring that the total exposure time does not exceed the maximum exposure time stated in Table 12.

\section{Sensitivity Estimation}

\subsection{Method to estimate the sensitivity}

The number of electrons generated from a point source emitting a flux $F_{\lambda}\left[\mathrm{W} / \mathrm{m}^{2} / \mu \mathrm{m}\right]$ is

$$
\begin{aligned}
S_{p} & =i_{p} \cdot t \\
& =\int_{\Delta \lambda} d \lambda \cdot F_{\lambda} \cdot\left(\frac{h c}{\lambda}\right)^{-1} \cdot A \cdot T_{\mathrm{sys}, p} \cdot \eta \cdot t .
\end{aligned}
$$

Here, $i_{p}[$ electrons $/ \mathrm{s}]$ is the total signal photo current emitted by the point source within the area

Table 10. Performances of detectors.

\begin{tabular}{lcc}
\hline & $\mathrm{Si}: \mathrm{As}^{\mathrm{a}}$ & $\mathrm{Si}^{\mathrm{S}} \mathrm{Sb}^{\mathrm{b}}$ \\
\hline Number of pixels & $2048 \times 2048$ & $1024 \times 1024$ \\
Pixel size $[\mu \mathrm{m}]$ & 25 & 18 \\
Dark current (SOAP) [electrons/s/pix] & 0.2 & 2.0 \\
Dark current (GOAL) [electrons/s/pix] & 0.03 & 0.4 \\
Available well depth (SOAP) [electrons] & $2.5 \mathrm{e}+05$ & $1.0 \mathrm{e}+05$ \\
Available well depth (GOAL) [electrons] & $2.5 \mathrm{e}+05$ & $1.0 \mathrm{e}+05$ \\
Read noise/sampling (SOAP) [electrons/sample] & 40 & 100 \\
Read noise/sampling (GOAL) [electrons/sample] & 30 & 100 \\
Read out pixel rate per one array [MHz] & 1 & 1 \\
Detective quantum efficiency & Fig. 2 in RCL2008 & Fig. 7 in KH2012
\end{tabular}

aThe SOAP data are measured typical values and the GOAL data are the measured best values for the JWST/MIRI array. These data are obtained from Love et al. (2006) and Ressler et al. (2008) (RCL2008 in the table).

${ }^{b}$ The SOAP dark current is scaled by size using the measured value in Van Cleve et al. (1995) for the Spitzer Si:Sb array, and the read noise is obtained from Mainzer et al. (2008) for the WISE detector which adopts the same read-out IC. Detective quantum efficiencies are taken from Khalap \& Hogue (2012) (KH2012 in this table and hereafter). The GOAL dark current is estimated as the sum of the measured dark current of the detector test device reported by KH201 and the possible dark current from the readout IC (private communication).

Table 11. Cosmic ray environment and maximum exposure time.

Acceptable fraction of hit pixel in an exposure

Cosmic ray hit event rate at L2 [events $/ \mathrm{m}^{2} / \mathrm{s}$ ]

Number of pixels affected by a single hit event

Maximum exposure time for $\mathrm{Si}: \mathrm{As}^{\mathrm{a}}$

Maximum exposure time for $\mathrm{Si}: \mathrm{Sb}^{\mathrm{a}}$

$$
\begin{gathered}
0.04 \\
5 \mathrm{e}+4 \\
4 \\
320.00 \\
617.28
\end{gathered}
$$

aThese values are derived from other values. 


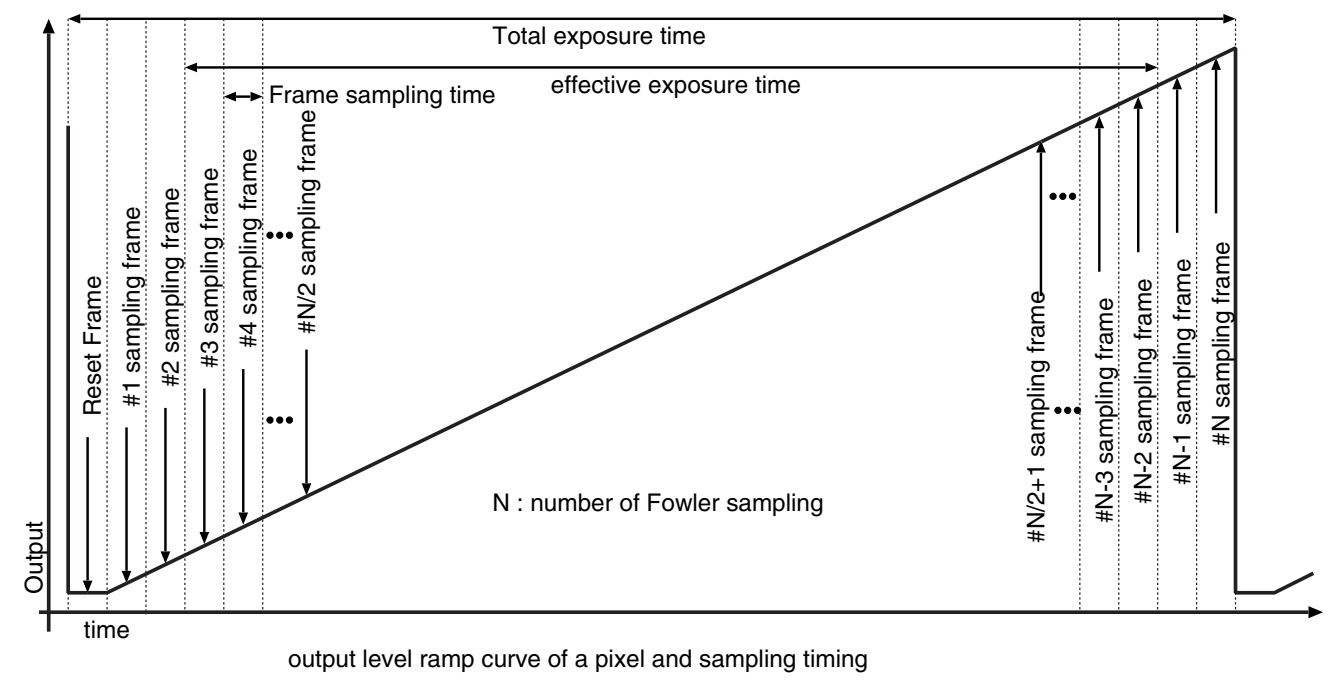

Fig. 8. Detector operation.

Table 12. Parameters of detector operation.

\begin{tabular}{|c|c|c|}
\hline & Si:As & $\mathrm{Si}: \mathrm{Sb}$ \\
\hline Number of frame cycles for long exposure & 72 & 571 \\
\hline Total exposure time $[\mathrm{s}]^{\mathrm{a}}$ & 301.99 & 598.74 \\
\hline Number of Fowler sampling & 16 & 16 \\
\hline Effective exposure time for long exposure $[\mathrm{s}]^{\mathrm{a}}$ & 264.24 & 589.30 \\
\hline Frame sampling time (shortest exposure) $[\mathrm{s}]^{\mathrm{a}}$ & 4.19 & 1.05 \\
\hline
\end{tabular}

${ }^{\text {a }}$ These values are derived from other values.

of aperture photometry, and $t[\mathrm{~s}]$ is the net integration time. $\Delta \lambda$ is the wavelength bandwidth, $A\left[\mathrm{~m}^{2}\right]$ is the effective area of the telescope, $\eta$ is the quantum efficiency of the detector, and $T_{\text {sys, } p}$ is the system throughput of the point source. $T_{\text {sys }, p}$ should include the energy fraction within the measurement area.

During imaging, the signal from a point source is evaluated by assuming an aperture photometry with radius $r_{\text {ap }}=1.22 \lambda / D$. On the other hand, in slit spectroscopy, we assume a rectangular area on the slit plane with a height of $2 \times 1.22 \lambda / D$ and a width defined by the slit. $T_{\text {sys }, p}$ should include the energy fraction of the point source intercepting the circle (rectangle area) on the detector plane of the imaging (slit spectroscopy). The former is called the encircled energy ratio, and the latter defines the slit efficiency for a point source.

The end-to-end system throughput consists of the telescope transmission $\left(T_{\mathrm{tel}}\right)$, efficiency of filter transmission and/or gratings $\left(T_{f}\right)$, encircled energy ratio within the aperture photometry of interest
$\left(T_{\text {ap }}\right)$, and transmission by other optical components in module $\mathrm{x}\left(T_{x}\right)$. Here, $\mathrm{x}$ is one of WFC-S/L, MRS$\mathrm{S} / \mathrm{L}$, and HRS. For a point source, because the optical quality of the system is nearly diffractionlimited, we only consider the Strehl ratio Sr, which includes the wavefront error (WFE) of the telescope, imperfections in the instruments and their alignment, and the end-to-end optical aberration in the design. The pointing error should also be considered. For simplicity, we incorporate the pointing error effect into $T_{\mathrm{ap}}$. The total throughput then becomes

$$
T_{\mathrm{sys}, p}=T_{\mathrm{tel}} \cdot T_{x} \cdot T_{f} \cdot \mathrm{Sr} \cdot T_{\mathrm{ap}} .
$$

We calculated the sensitivities for various sources assuming that $\lambda F_{\lambda}$ is constant across the wavelength region. This convention has been adopted by other satellites, namely, IRAS, COBE, ISO, Spitzer/IRAC, and AKARI IRC. The photo current in Eq. (7) is now calculated as

$$
i_{p}=\frac{\lambda_{c} F_{\lambda_{c}}}{h c} \cdot A \cdot \int_{\lambda_{s}}^{\lambda_{l}} d \lambda \cdot T_{\mathrm{sys}, p}(\lambda) \cdot \eta(\lambda) .
$$

Here, $\lambda_{c}, \lambda_{s}$, and $\lambda_{l}$ denote the central, shortest, and longest wavelengths in the band, respectively. For simplicity, we assume that $\mathrm{Sr}$ and $T_{\text {ap }}$ are independent of wavelength; that is

$$
\begin{aligned}
i_{p}= & \frac{\lambda_{c} F_{\lambda_{c}}}{h c} \cdot A \cdot \operatorname{Sr}\left(\lambda_{c}\right) \cdot T_{\mathrm{ap}}\left(\lambda_{c}\right) \\
& \times \int_{\lambda_{s}}^{\lambda_{l}} d \lambda \cdot T_{\mathrm{tel}}(\lambda) \cdot T_{x}(\lambda) \cdot T_{f}(\lambda) \cdot \eta(\lambda) .
\end{aligned}
$$


The number of noise electrons $N_{p}$ for a point source is given by

$$
\begin{aligned}
N_{p}^{2}= & \left(i_{p}+i_{b g} \cdot n+i_{\mathrm{det}} \cdot n\right) \cdot t+N_{\mathrm{read}}^{2} \cdot n \\
& \cdot n_{\mathrm{frame}} \cdot
\end{aligned}
$$

Here, $i_{b q}[$ electrons/s] is the photo current per pixel generated by the background radiation, $n$ is the number of pixels used in the photometry, $i_{\text {det }}$ [electrons/s] is the detector dark current per pixel, $N_{\text {read }}$ is the readout noise per pixel in each frame, and $n_{\text {frame }}$ is the number of frames within the net integration time $t$.

Let $t_{\mathrm{obs}}, t_{f}$, and $t_{e}$ be the observing, frame, and effective exposure times, respectively, for a given frame. Then we have

$$
n_{\text {frame }}=\frac{t_{\mathrm{obs}}}{t_{f}}=\frac{t}{t_{e}} .
$$

The $\mathrm{S} / \mathrm{N}$ ratio for a point source is defined as $\mathrm{SNR}=S_{p} / N_{p}$. We estimate system sensitivity to a source flux with SNR $=5$ over $t_{\text {obs }}=3600 \mathrm{~s}$ (time integration of $1 \mathrm{hr}$ ).

\subsection{Errors affecting the throughput and sensitivity}

Within the total error budget, we consider two sources of WFEs and the effect of pointing error. First, we consider the effect of the WFEs on the point spread function (PSF), and then calculate the effect of the pointing error.

\subsubsection{Effect of WFEs on PSF}

The PSF is assumed to be sourced from (1) diffraction limited PSF with central obscuration, and (2) a widespread component. It is formulated as

$$
\begin{aligned}
\operatorname{PSF}(r, \theta)= & \operatorname{Sr} \cdot \frac{\left|J_{1}(\pi r) / 2 r-d \cdot J_{1}(\pi r d) / 2 r\right|^{2}}{\pi / 4\left(1-d^{2}\right)} \\
& +(1-\mathrm{Sr}) \cdot P(r, \theta)_{\text {widespread }} .
\end{aligned}
$$

Here, Sr is the Strehl ratio, $r$ and $\theta$ are the polar coordinates in the focal plane normalized by $\lambda / D, d$ is the radius ratio of the central obscuration to the entrance pupil diameter (EPD), and $J_{1}$ is the Bessel function. The total energy, obtained by integrating the PSF over $r$ and $\theta$, is normalized to 1 . The Strehl ratio is defined as the peak intensity of a point source over the diffraction-limited intensity imposed by the system's entrance aperture. Defined in this manner, the Strehl ratio is not reduced by central obscuration.
The second term $(1-\mathrm{Sr}) \cdot P_{\text {widespread }}$ reduces the energy given by the first term when $\mathrm{Sr}<1$. We assume that MCS/SPICA is nearly diffraction-limited and that the WFEs are not concentrated at the few lowest spatial frequencies. Under these conditions, the energy from the point source distributes more widely, over the first null ring $(r=1.22 \lambda / D)$ of the diffraction-limited PSF of a circular entrance aperture. Thus, the second term is negligible (relative to the first term) within an area of radius $1.22 \lambda / D$, the area of the aperture photometry. The Strehl ratio Sr in Eq. (8), will be introduced to the total throughput later. The WFEs then enter the estimation of $\mathrm{Sr}$ and the effect of pointing error is estimated from the diffraction-limited PSF.

The first of the WFE sources is the SPICA telescope assembly (STA) error, which includes the errors in manufacturing, assembly and integration, cool-down, and distortion at launch and in space. The STA error excludes optical aberration in the telescope optical design. Because the FOV of MCS is wide and distant from the optical center of the telescope, large optical aberration is unavoidable. This aberration should be partially compensated by the optical design of the focal plane instrument (FPI) such as MCS. The remaining aberration should be included in the WFE of FPI optics. Along with the FPI, the second component of the WFE contains the errors at the STA-FPI interface. This component embodies the designed total optical aberration, imperfections in the FPI optics (introduced by manufacturing, assembly, integration, and other factors), co-focal misalignment of the FPIs, and misalignment of the optical reference between the STA and FPIs; although this last factor will be alleviated by secondary adjustment mechanism on the telescope.

According to the preliminary studies of STA conducted by industrial institutes, the WFE in STA is approximately $240 \mathrm{~nm}$. Here, we conservatively allocate $250 \mathrm{~nm}$ to the WFE of STA. In our preliminary design and tolerance analysis of MCS, the worstcase Strehl ratio was approximately 0.8 at $5 \mu \mathrm{m}$. Therefore, in the worst-case scenario, the second WFE in the above-mentioned allocation is approximately $350 \mathrm{~nm}$. The reducing factor introduced by pointing instability is allocated as 0.9 , giving the same effect as the WFE of STA at $5 \mu \mathrm{m}$. As discussed in a later section, the reducing factor induced by pointing instability and the estimated RMS in the pointing angle error require adjustment by an adaptive system with a TTM at short wavelengths. 
The WFE in the Strehl ratio $\left(\sigma_{\mathrm{WFE}}\right)$ is computed using the Marechal formula

$$
\mathrm{Sr}=1-\left(2 \pi \frac{\sigma_{\mathrm{WFE}}}{\lambda}\right)^{2}
$$

where

$$
\sigma_{\mathrm{WFE}}^{2}=\sigma_{\mathrm{STA}}^{2}+\sigma_{\mathrm{FPI}}^{2} .
$$

The Strehl ratio calculated by the Marechal approximation is recognized to be slightly worse than the true ratio (Mahajan, 1983).

\subsubsection{Effects of the pointing error}

In an analysis of SPICA's pointing control system (Mitani et al., 2014), two potential noise sources were identified in the pointing error. The first is vibrations at mid-frequencies (between 0.03 and $\sim 1 \mathrm{~Hz}$ ). Below $0.03 \mathrm{~Hz}$, i.e. within the bandwidth of the attitude control system, the pointing is controllable. Higher frequency vibrations (above $\sim 1 \mathrm{~Hz}$ ) are measurable and controllable and do not affect the pointing, as a result of spacecraft model analysis. The mid-frequency region is problematic because the pointing system is exposed to vibrations without active control. Any unexpected resonances and vibration distributions in this frequency region must therefore be attenuated. As a conservative estimate, we assume the error as the limit of vibration measurements on the ground. The second potential noise, attitude disturbance, was observed in a previous mission called HINODE. From the dynamics and attitude control model of SPICA, we derived the power spectrum density (PSD) of the angular error. The PSD of the pointing angle error is plotted in Fig. 9.

To estimate the reducing factor associated with the pointing error, we require the pointing angle distribution function rather than the PSD. We assume that a sufficient number of independent noise sources contribute to the PSD. From the central limit theorem, the pointing angle distribution function is then expressed in a Gaussian form. If the pointed angle rotates around the target point, the

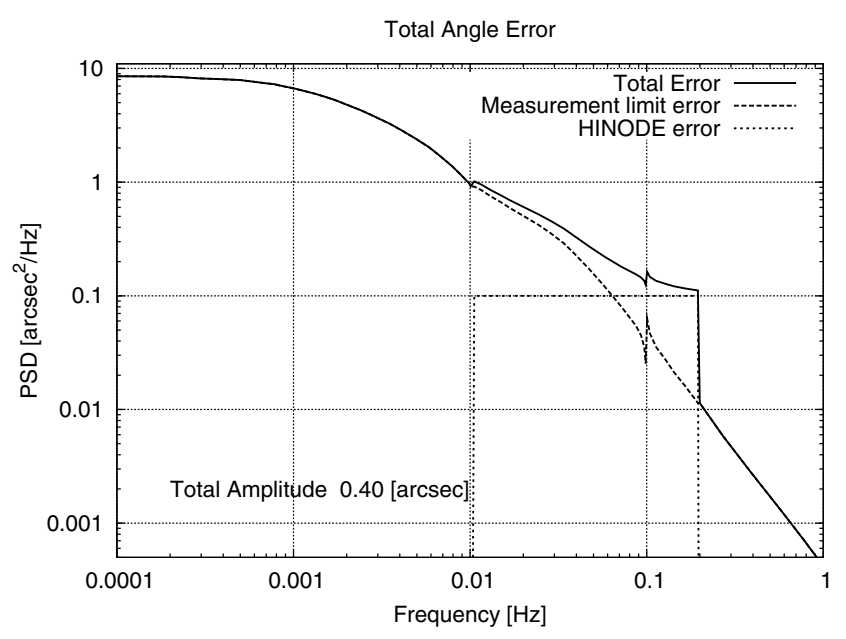

Fig. 9. Estimated PSD of the pointing error. The measurement limit error and the HINODE error are defined in the text.

pointing error is largest for a particular distribution of pointing angles. In this case, the pointing dispersion at each frequency is given as the amplitude $\sqrt{\operatorname{PSD}(f) \times \delta f}$. Thus, the distribution function is assumed as

$$
P(x, y)=\frac{1}{2 \pi \sigma_{p}^{2}} \exp \left(-\frac{x^{2}+y^{2}}{2 \sigma_{p}^{2}}\right),
$$

where

$$
\sigma_{p}^{2}=\int \operatorname{PSD}(f) d f
$$

The adopted value of $\sigma_{p}$ is given in Table 13 .

In the absence of pointing error, the encircled energy ratio gives the throughput of the imaging as

$$
\begin{aligned}
T_{\text {ap }, 0} & =E_{c}\left(r_{\text {ap }}\right) \\
& =\int_{0}^{r_{\text {ap }}} 2 \pi r \operatorname{PSF}(r) d r .
\end{aligned}
$$

Note that the PSF defined by Eq. (13) gives $E_{c}(\infty)=1$. Introducing an instantaneous pointing error $\rho$ into the energy ratio, we obtain

$$
E_{c}\left(r_{\mathrm{ap}}, \rho\right)=\int^{|\vec{x}| \leq r_{\mathrm{ap}}} \operatorname{PSF}(x-\rho, y) d x d y .
$$

Table 13. Allocated WFEs, reducing factor introduced by pointing error and Strehl ratio.

\begin{tabular}{lc}
\hline Allocated RMS WFE of STA $[\mathrm{nm}] \sigma_{\mathrm{STA}}$ & 250 \\
Allocated RMS WFE of FPI and alignment between STA and FPI $[\mathrm{nm}] \sigma_{\mathrm{FPI}}$ & 350 \\
Allocated reducing factor by pointing instability & 0.90 \\
Estimated RMS pointing angle error $\sigma_{p}[\operatorname{arcsec}]$ & 0.40 \\
\hline
\end{tabular}


Because the distribution function is Gaussian and PSF is rotationally symmetric, the encircled energy ratio with pointing error becomes

$$
\begin{aligned}
\left\langle E_{c}\left(r_{\text {ap }}\right)\right\rangle_{\sigma_{p}}= & \int E_{c}\left(r_{\text {ap }}, \rho\right) \frac{\rho}{\sigma_{p}^{2}} \\
& \times \exp \left(-\frac{\rho^{2}}{2 \sigma_{p}^{2}}\right) d \rho .
\end{aligned}
$$

The reducing factor $\zeta$ and throughput are given by

$$
\zeta_{\mathrm{ap}}=\frac{\left\langle E_{c}\left(r_{\mathrm{ap}}\right)\right\rangle_{\sigma_{p}}}{E_{c}(r, 0)}
$$

and

$$
T_{\text {ap }}=\left\langle E_{c}\left(r_{\text {ap }}\right)\right\rangle_{\sigma_{p}} .
$$

Figure 10 shows how the reducing factor $\zeta$ depends on the pointing error $\sigma_{p}$. At wavelengths shorter than $12.4 \mu \mathrm{m}$ and $\sigma_{p}=0.4$ arcsec, the pointing error must be compensated by a TTM to achieve $\zeta<0.9$. As shown in Fig. 3, the TTM is installed in the fore-optics of the WFC-S and HRS modules.

In slit spectroscopy, we consider the pointing error in a rectangle area with height $h=2 \times$ $1.22 \lambda / D$ and full width $w$, corresponding to the slit width. In the absence of pointing error, the throughput is given by

$$
\begin{aligned}
T_{\text {slit }, 0} & =E_{r}(h, w) \\
& =\int_{-h / 2}^{h / 2} d y \int_{-w / 2}^{w / 2} d x \operatorname{PSF}(x, y) .
\end{aligned}
$$

Here, the slit efficiency $T_{\text {slit }}$ replaces $T_{\text {ap }}$ in Eq. (10). By introducing an instantaneous pointing error $\rho_{x}$,

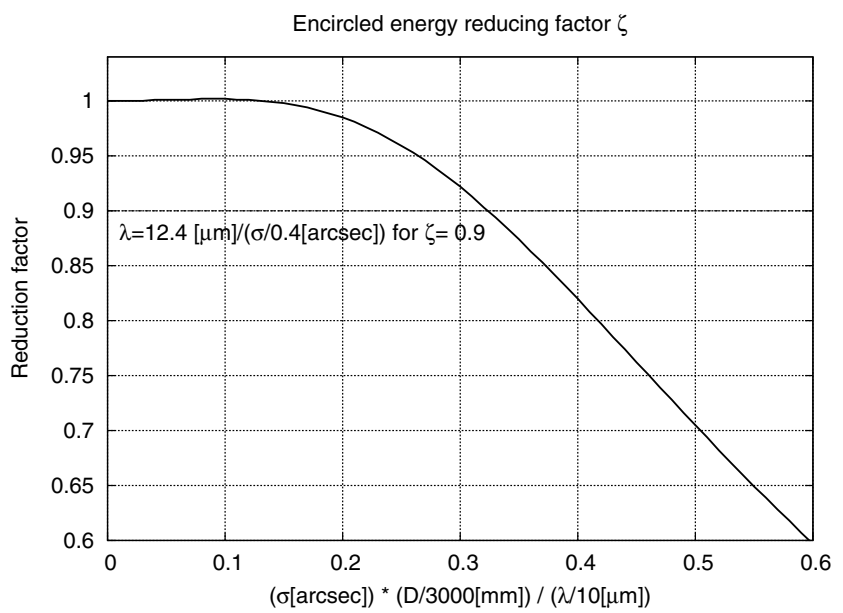

Fig. 10. Reduction factor vs. pointing error normalized by $\lambda / D$. $\rho_{y}$, the energy ratio in the rectangular area becomes

$$
\begin{aligned}
& E_{r}\left(h, w, \rho_{x}, \rho_{y}\right) \\
& \quad=\int_{-h / 2}^{h / 2} d y \int_{-w / 2}^{w / 2} d x \operatorname{PSF}\left(x-\rho_{x}, y-\rho_{y}\right) .
\end{aligned}
$$

Then, we have

$$
\begin{aligned}
T_{\text {slit }}= & \left\langle E_{r}(h, w)\right\rangle_{\sigma_{p}} \\
= & \int E_{r}\left(h, w, \rho_{x}, \rho_{y}\right) \frac{1}{2 \pi \sigma_{p}^{2}} \\
& \times \exp \left(-\frac{\rho_{x}^{2}+\rho_{y}^{2}}{2 \sigma_{p}^{2}}\right) d \rho_{x} d \rho_{y} .
\end{aligned}
$$

Figure 11 shows the reducing factors and slit efficiencies of the MRS-S, MRS-L, and HRS modules. Clearly, HRS requires a compensatory TTM system.

The temperature of the SPICA telescope is maintained at approximately $6 \mathrm{~K}$ by passive and active cooling. Active cooling is performed by mechanical coolers with two thermal stages at base temperatures of $4.5 \mathrm{~K}$ and $1.7 \mathrm{~K}$. The FPI assembly is maintained at about $4.5 \mathrm{~K}$. Thermal emissions from the telescope and instrument are negligible at mid-infrared wavelengths, and the instrument background is dominated by zodiacal light. This background emission is modeled by blackbody radiation at an equivalent temperature and a normalization factor based on some reference wavelength. We consider both high and low background conditions. Under

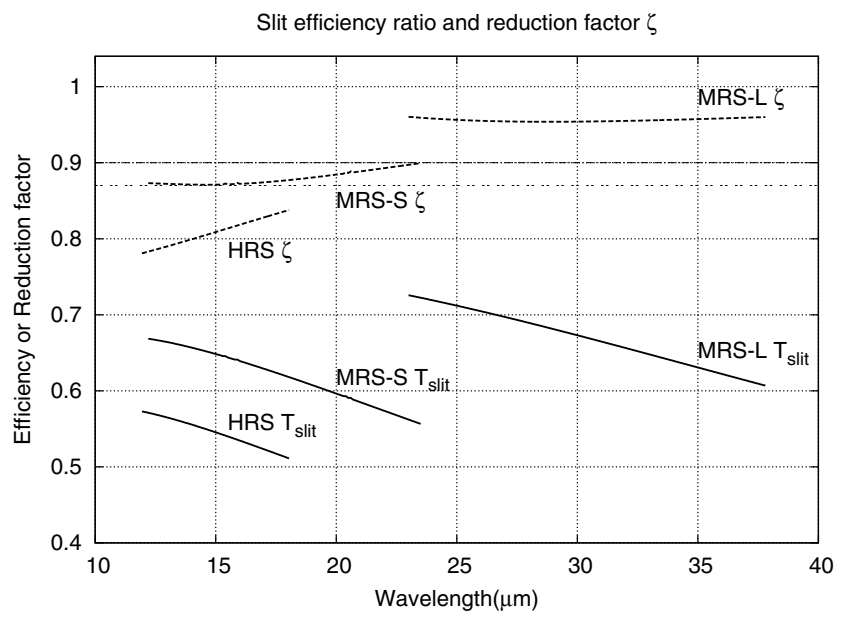

Fig. 11. Reduction factor $\zeta$ of slit efficiency in MRS-S, MRS-L, and HRS under the conditions listed in Table 13. $\zeta$ for MRS-S lie between 0.87 and 0.90 , slightly beyond the requirements of Table 13 but acceptable. On the other hand, $\zeta$ for HRS is smaller than the allocated value and must be corrected by a TTM system. 
Table 14. Background flux.

High background case black body temperature $[\mathrm{K}] \quad 268.5$

High background case reference wavelength $[\mu \mathrm{m}] \quad 25$

High background case normalization flux [MJy/Sr] 80

Low background case black body temperature $[\mathrm{K}] \quad 274.0$

Low background case reference wavelength $[\mu \mathrm{m}] \quad 25$

Low background case normalization flux [MJy/Sr] 15

high background conditions, we assume that flux condition at the ecliptic plane at $\beta=0.0^{\circ}$ and $\epsilon=60^{\circ}$; in the low background case, flux condition at the ecliptic pole at $\beta=90^{\circ}$. The parameters given in Table 14 are obtained from the observational results of ISO and IRTS (Reach et al., 2003; Ootsubo et al., 2000).

\subsection{Sensitivities of $M C S$}

\subsubsection{Throughputs}

As shown in Eq. (10), the signal current is proportional to the product of several factors: $\mathrm{Sr} \cdot T_{\text {ap }}$. $T_{\text {tel }} \cdot T_{x} \cdot T_{f} \cdot \eta$. In slit spectroscopy, the $T_{\text {ap }}$ term denoting the encircled energy ratio within an area, which is used in aperture photometry, is replaced by the slit efficiency $T_{\text {slit }}$. The effects of individual components on the throughputs of WFC, MRS, and HRS are plotted in Figs. 12-14, respectively. Figure 15 shows the total throughputs of MCS.

The encircled energy ratio within the radius $1.22 \lambda / D$ decreases with decreasing wavelength.

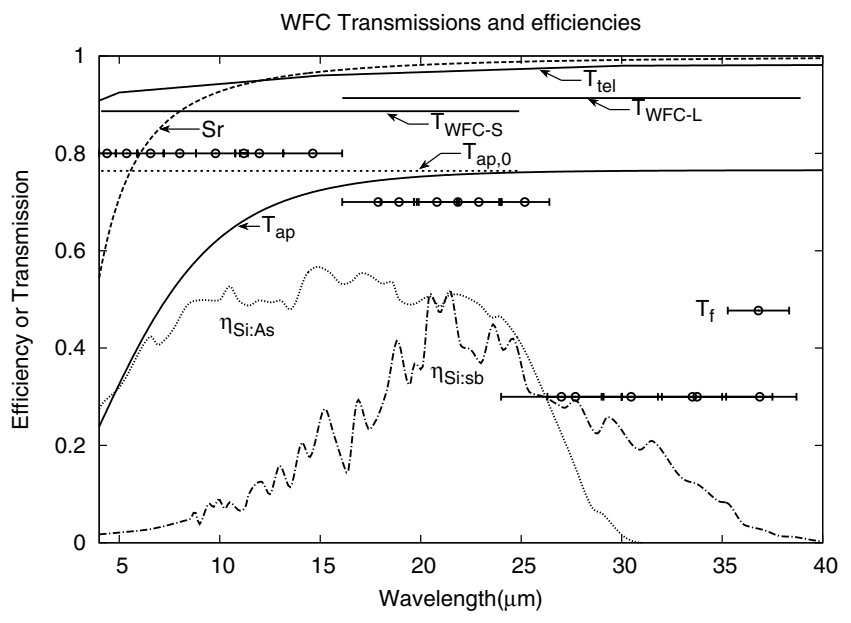

Fig. 12. Wavelength dependences of the factors affecting WFC throughput. The parameters $\mathrm{Sr}, T_{\mathrm{ap}}, T_{\mathrm{tel}}, T_{\mathrm{WFC}-S}$, $T_{\mathrm{WFC}-L}, T_{f}$, and $\eta$ are those used in Eq. (4.1). $T_{\text {ap }, 0}$ and $T_{\text {ap }}$ are the encircled energy ratios in the absence and presence of pointing error, respectively. The detective quantum efficiencies are obtained from Ressler et al. (2008) and Khalap \& Hogue (2012).

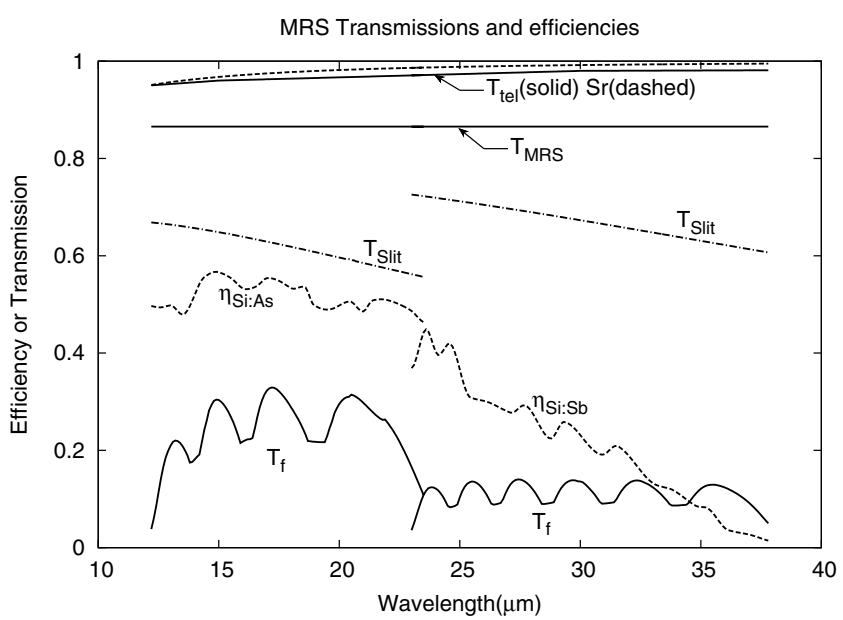

Fig. 13. Wavelength dependences of the factors affecting MRS throughput. $T_{f}$ denotes the total efficiency of the echelle grating, cross disperser, dichroic beam splitter, and BPF. $T_{\text {slit }}$ is the slit efficiency defined by Eq. (25). The detective quantum efficiencies are obtained from Ressler et al. (2008) and Khalap \& Hogue (2012).

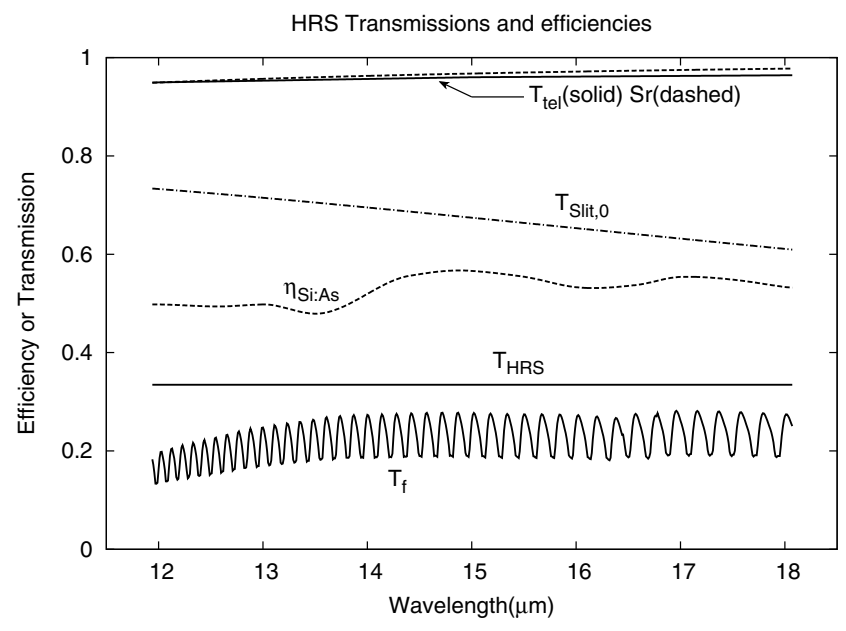

Fig. 14. Wavelength dependences of the factors affect HRS throughput. $T_{f}$ includes the efficiencies of the echelle grating, cross disperser, $\mathrm{BPF}$, and transmission of the immersion grating. $T_{\text {slit }, 0}$ is the slit efficiency without pointing error.

This degradation can be compensated by an adaptive system using TTM.

Remarkably, at long wavelengths, the throughputs of the BPFs are low (see Sec. 3.2) and the efficiency of the $\mathrm{Si}: \mathrm{Sb}$ detector is reduced. In the MRS-L module, the $T_{f}$ is reduced by the low transmissions of the dichroic beam splitter and BPF. Compared with the MRS optics transmissions $T_{\mathrm{MRS}-S}$ and $T_{\mathrm{MRS}-L}$, the HRS optics transmission $T_{\mathrm{HRS}}$ is relatively low because of the lens surface transmissions. 


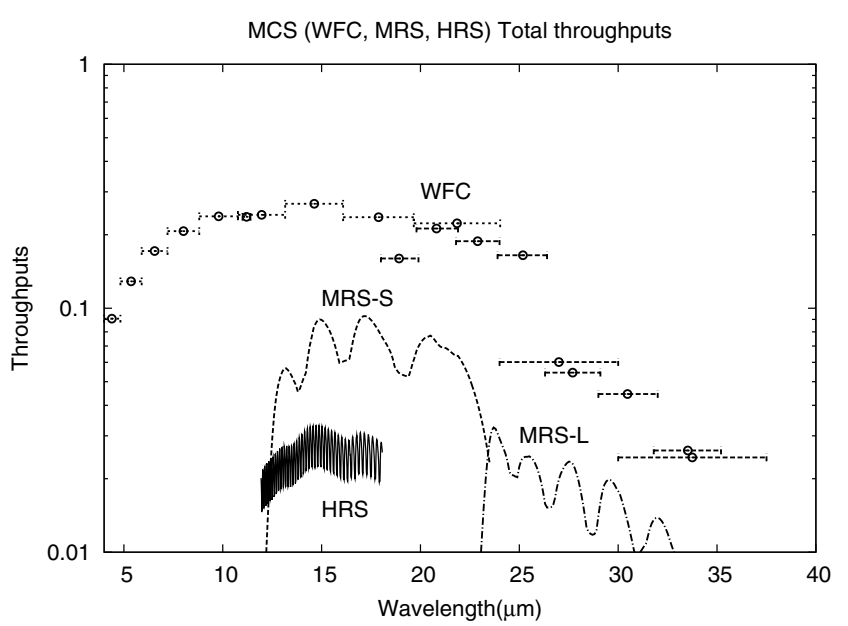

Fig. 15. Throughput of WFC, MRS, and HRS. The points with horizontal dotted (dashed) bars indicate the total throughputs of WFC-S (WFC-L). We applied a tip-tilt correction to WFC-S and HRS, but no correction to WFC-L, MRS-S, and MRS-L. The dashed, dot-dashed, and continuous lines show the total throughputs of MRS-S, MRS-L, and HRS, respectively.

\subsubsection{Noise contributions}

Figures 16-18 plot the noise from the background zodiacal emission $\left(\sqrt{i_{b g} \cdot n}\right)$, detector dark current noise $\left(\sqrt{i_{\text {det }} \cdot n}\right)$, and readout noise with Fowler sampling $\left(N_{\text {read }} \cdot \sqrt{n / t_{e}}\right)$, respectively, as functions of wavelength.

Noise in WFC is mainly sourced from background zodiacal emissions; the dark current and readout noise in the detector are almost negligible.

Similarly, the noise in the MRS-S module chiefly comprises background zodiacal emission; on the other hand, the MRS-L module is subject to background emissions and the SOAP detector noise in roughly equal quantities. The noise in the HRS

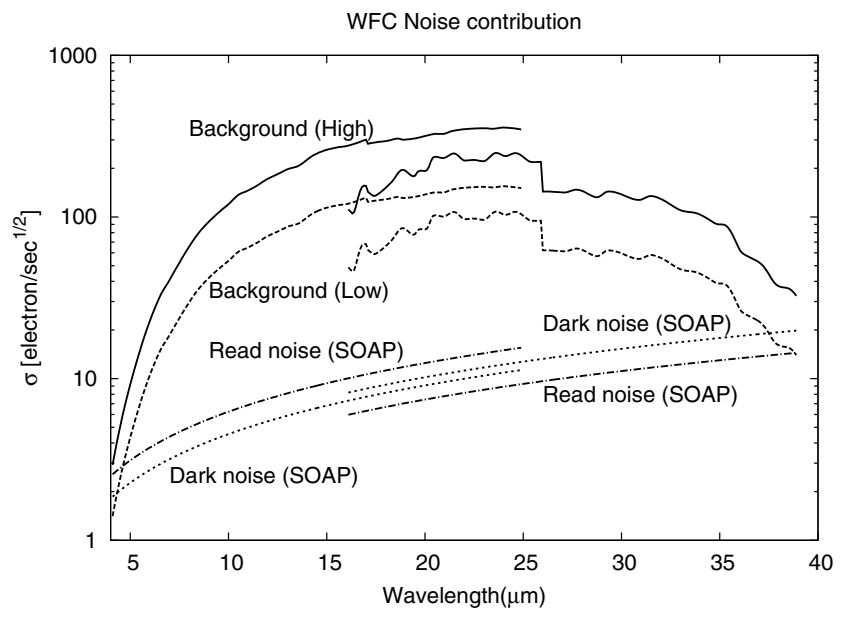

Fig. 16. Contributions of noise components in WFC.

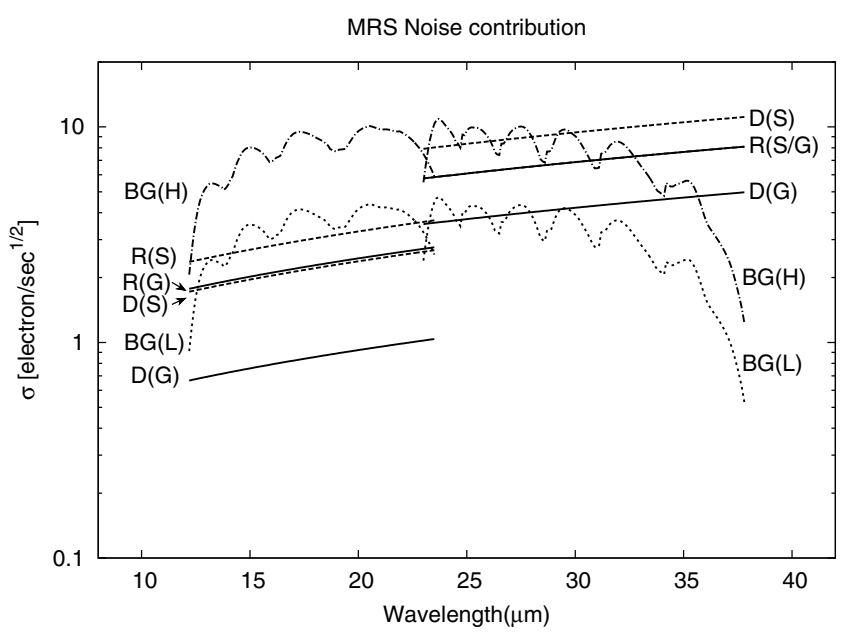

Fig. 17. Contributions of noises in MRS. Dark current noises in the SOAP and GOAL detectors (see Table 10) are denoted D (S) and $\mathrm{D}(\mathrm{G})$, respectively. The corresponding readout noises are indicated as $\mathrm{R}(\mathrm{S})$ and $\mathrm{R}(\mathrm{G})$. High and low background noises are denoted by $\mathrm{BG}(\mathrm{H})$ and $\mathrm{BG}(\mathrm{L})$, respectively.

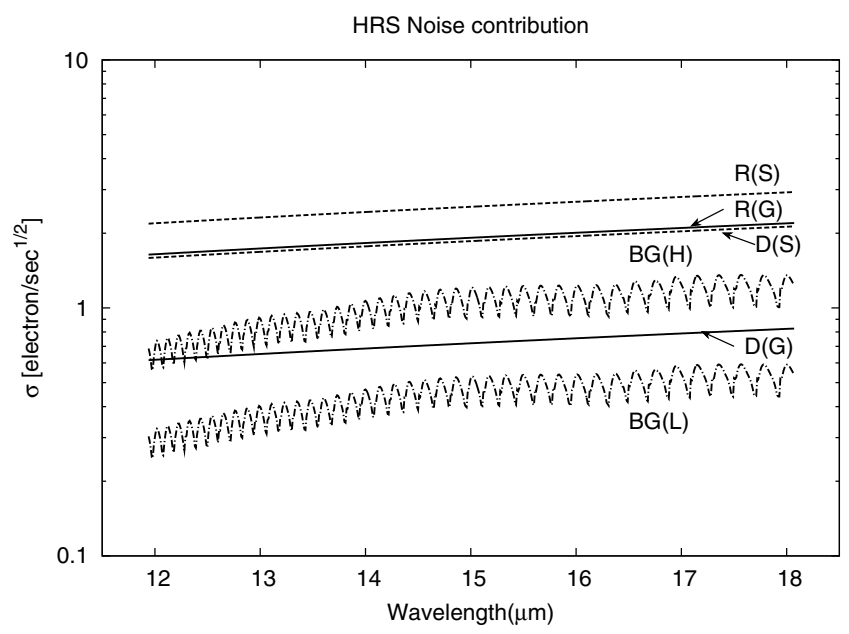

Fig. 18. Contributions of noises in HRS. The notations are defined in the caption of Fig. 17.

module is dominated by detector noise, even under the GOAL detector performance.

\subsubsection{Limiting point source flux and saturation limit}

Point source fluxes corresponding to $\mathrm{SNR}=5$ over $1 \mathrm{hr}$ observing duration are plotted in Figs. 19-21. Figure 22 plots the line detection limit in the MRS and HRS modules.

Figure 23 shows the point source fluxes that saturate the WFC module within the minimum exposure time with Fowler 1 sampling. Here, pointing error is ignored because of the short exposure time. The ratio of the saturation limit to the 


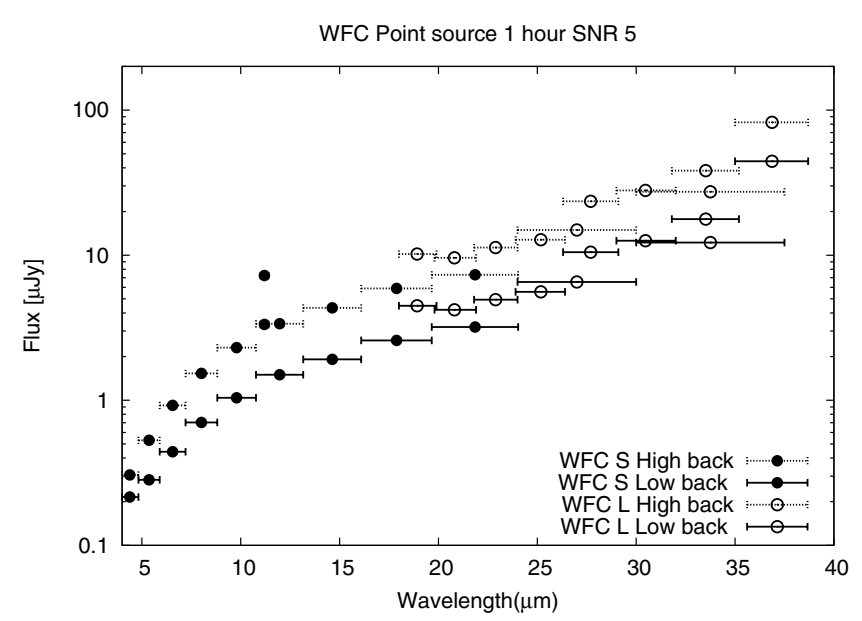

Fig. 19. WFC imaging sensitivity for a point source corresponding to a source observed for $1 \mathrm{hr}$ on source with $\mathrm{SNR}=5$. The sensitivity at each band is specified for each filter in Table 4. The horizontal bars show the bandwidths. Both high background case low background cases defined in Table 14 are shown. The pointing error corrected by TTN in WFC-S but not in WFC-L.

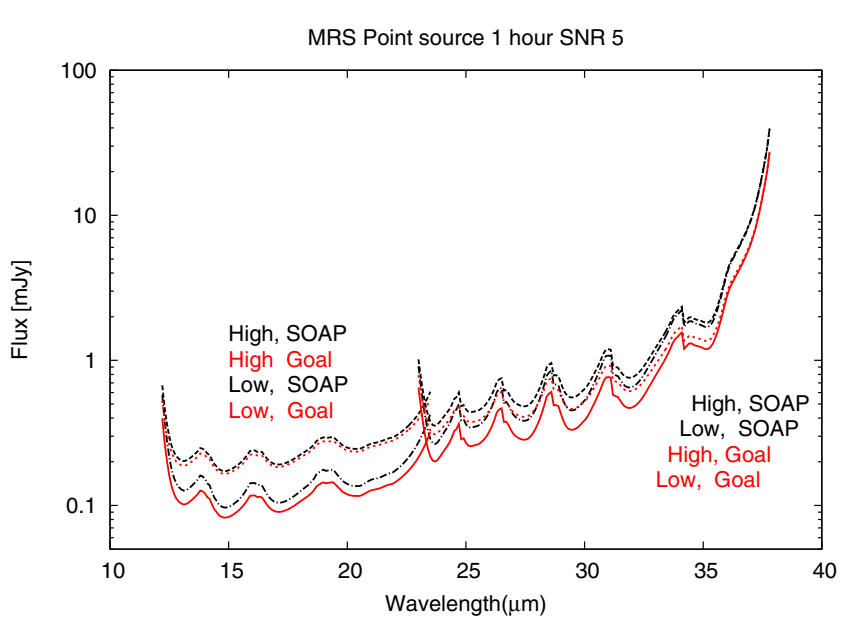

Fig. 20. Point source fluxes per spectral resolution element corresponding to a source observed for $1 \mathrm{hr}$ on source with $\mathrm{SNR}=5$ by MRS. The continuous, dotted, dot-dashed, and dashed curves plot the cases of low background condition and GOAL detector, high background and GOAL, low background and SOAP, and high background and SOAP, respectively.

detection limit is approximately $3 \times 10^{5}$ in both the MRS and HRS modules, indicating saturation limits of a few tens Jy or higher.

\section{Key Factors to get High Performances}

The pointing error, estimated as $\sigma_{p}=0.4$ arcsec, is chiefly contributed by the measurement limit of the

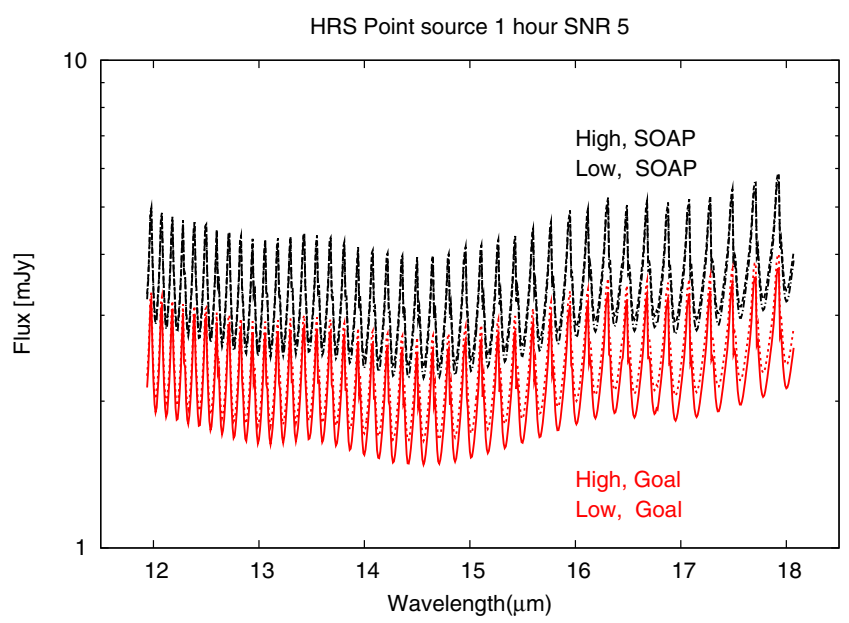

Fig. 21. Point source fluxes per spectral resolution element corresponding to a source observed for $1 \mathrm{hr}$ on source with $\mathrm{SNR}=5$ by HRS. The notations are defined in the caption of Fig. 20. The pointing error was corrected by TTM.

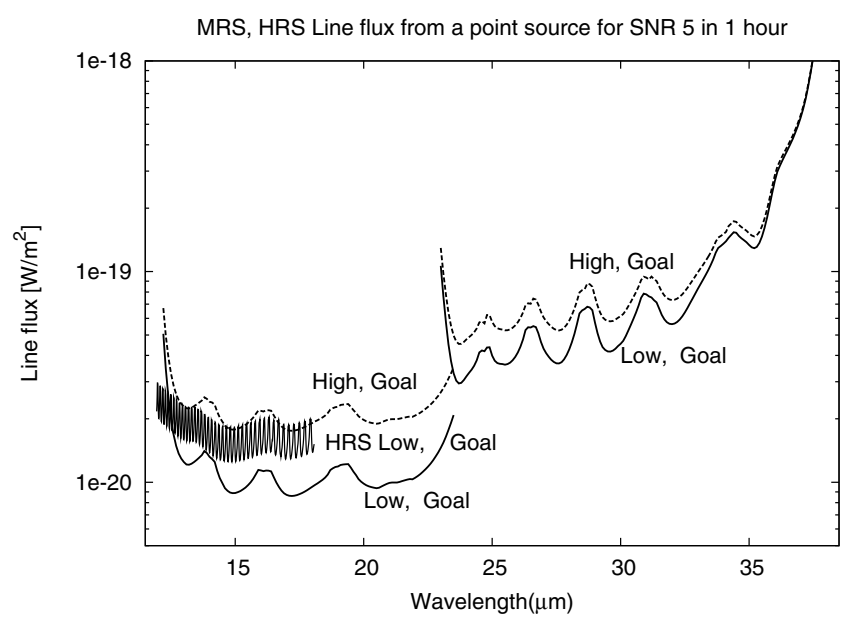

Fig. 22. Unresolved line fluxes during $1 \mathrm{hr}$ observation of a point source with $\mathrm{SNR}=5$. A GOAL detector was assumed. The continuous and dashed curves show the high and low background condition, respectively.

vibrations prior to launch. Conceivably, we may extend the measurement limit, but here we conservatively assume the worst-case scenario. As shown in Figs. 10 and 11, WFC-S and HRS require pointing error correction at short wavelengths (below $12.4 \mu \mathrm{m}$ ). Therefore, we install a TTM in the fore-optics of both the modules. MRS-S is more efficient than HRS over the same wavelength range because the slit width is wider in MRS-S than in HRS. However, the reduction factor of MRS-S is slightly below the requirement (see Fig. 11). To improve the efficiency of MRS-S, we can adopt a 


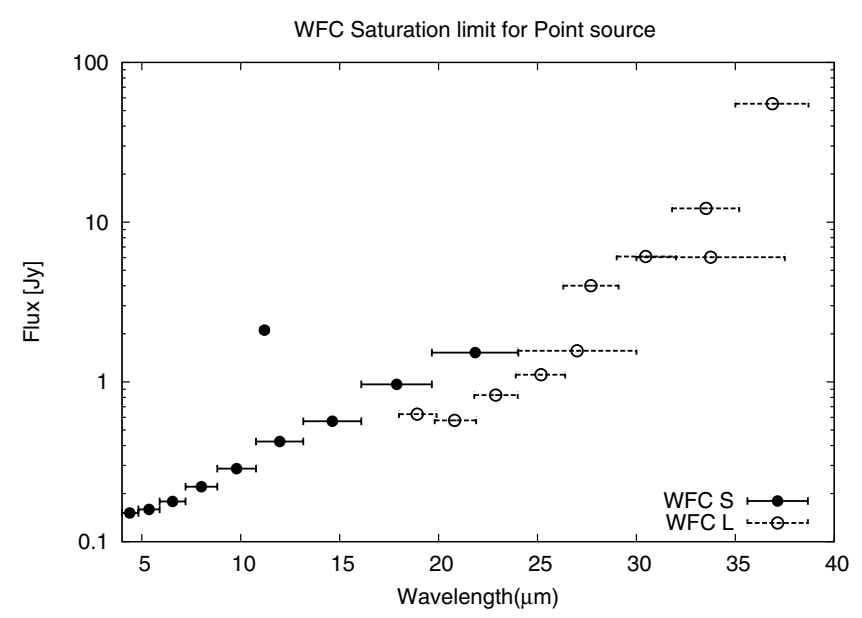

Fig. 23. Saturation limits of point source observation by WFC at each wavelength band. Horizontal bars indicate the bandwidths.

slightly wider slit but this would compromise the resolving power.

As mentioned in Sec. 3.2, the BPFs are deficient at longer wavelengths. The BPF transmission in the wavelength region $\lambda_{c} \geq 26 \mu \mathrm{m}$ was assumed to be $30 \%$. In a trial production of a dichroic beam splitter for MRS, the transmission to MRS-L was approximately $30 \%$, which is the same as the abovementioned value. At longer wavelengths, the thickness of each BPF layer must be increased. This is problematic considering the very limited choice of materials that are transparent to these wavelengths. Therefore, to improve the total throughputs of WFC-L and MRS-L, we must develop much more transparent BPFs than those currently available.

Grating efficiency is another cause of low throughput. The adjustment factors in Tables 6 and 8 can be explained by the effect of phase shift on the metal surface and multiple diffractions within a grating groove (Palner \& Lebrun, 1972). Consequently, the efficiency depends not only on the reflecting surface of a groove but also on the nonilluminated surface. The groove shape can be optimized by electromagnetic wave analysis. Because the gratings are manufactured by ultra-high precision cutting machines, we can fabricate the optimal

Table 15. Performances of MCS instruments.

\begin{tabular}{|c|c|c|c|c|c|c|c|c|}
\hline Module & & \multicolumn{3}{|l|}{ WFC-S } & \multicolumn{4}{|l|}{ WFC-L } \\
\hline Wavelength $[\mu \mathrm{m}]$ & & \multicolumn{3}{|l|}{$5.0-24$} & \multicolumn{4}{|l|}{$18-38$} \\
\hline $\mathrm{FoV}$ & & \multicolumn{3}{|l|}{$5^{\prime} \times 5^{\prime}$} & \multicolumn{4}{|l|}{$5^{\prime} \times 5^{\prime}$} \\
\hline Array format & & \multicolumn{3}{|l|}{$2 \mathrm{k} \times 2 \mathrm{k}$} & \multicolumn{4}{|l|}{$1 \mathrm{k} \times 1 \mathrm{k}$} \\
\hline Spectral resolution & & \multicolumn{3}{|l|}{5} & \multicolumn{4}{|l|}{10} \\
\hline \multicolumn{9}{|l|}{ Sensitivity ${ }^{\mathrm{a}}$} \\
\hline Wavelength $[\mu \mathrm{m}]$ & 5.4 & 12 & 22 & 25 & 30 & 34 & & \\
\hline High background case $(\mu \mathrm{Jy})$ & 0.53 & 3.4 & 7.3 & 13 & 28 & 38 & & \\
\hline Low background case $(\mu \mathrm{Jy})$ & 0.28 & 1.5 & 3.2 & 5.6 & 13 & 18 & & \\
\hline Module & \multicolumn{5}{|c|}{ MRS-S } & \multicolumn{3}{|c|}{ MRS-L } \\
\hline Wavelength $[\mu \mathrm{m}]$ & \multicolumn{4}{|c|}{$12-24$} & \multicolumn{4}{|c|}{$23-38$} \\
\hline FoV & \multicolumn{4}{|c|}{$12^{\prime \prime} \times 8^{\prime \prime}$} & \multicolumn{4}{|c|}{$12^{\prime \prime} \times 8^{\prime \prime}$} \\
\hline Array format & \multicolumn{4}{|c|}{$2 \mathrm{k} \times 2 \mathrm{k}$} & \multicolumn{4}{|c|}{$1 \mathrm{k} \times 1 \mathrm{k}$} \\
\hline Spectral resolution & \multicolumn{4}{|c|}{$1800-1950$} & \multicolumn{4}{|c|}{$720-1000$} \\
\hline \multicolumn{9}{|l|}{ Sensitivity ${ }^{\mathrm{b}}$} \\
\hline Wavelength $[\mu \mathrm{m}]$ & 13.8 & 14.8 & 19.0 & 20.5 & 24.7 & 25.2 & 34.1 & 35.1 \\
\hline High background case (mJy) & 0.23 & 0.16 & 0.28 & 0.24 & 0.49 & 0.37 & 1.7 & 1.4 \\
\hline Low background case (mJy) & 0.13 & 0.083 & 0.14 & 0.12 & 0.37 & 0.26 & 1.6 & 1.2 \\
\hline Module & \multicolumn{8}{|c|}{ HRS } \\
\hline Wavelength $[\mu \mathrm{m}]$ & \multicolumn{8}{|c|}{$12-18$} \\
\hline FoV & \multicolumn{8}{|c|}{$6^{\prime \prime} \times 1.2^{\prime \prime}$} \\
\hline Array format & \multicolumn{8}{|c|}{$2 \mathrm{k} \times 2 \mathrm{k}$} \\
\hline Spectral resolution & \multicolumn{8}{|c|}{$22,000-30,000$} \\
\hline \multicolumn{9}{|l|}{ Sensitivity ${ }^{\mathrm{b}}$} \\
\hline Wavelength $[\mu \mathrm{m}]$ & 12.50 & 12.54 & 14.96 & 15.01 & 17.49 & 17.57 & & \\
\hline High background case (mJy) & 3.1 & 1.9 & 2.8 & 1.7 & 3.7 & 3.5 & & \\
\hline Low background case (mJy) & 3.0 & 1.7 & 2.7 & 1.5 & 2.2 & 2.0 & & \\
\hline
\end{tabular}

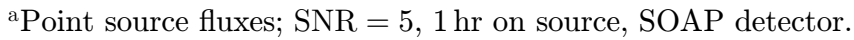

${ }^{\mathrm{b}}$ Point source fluxes; $\mathrm{SNR}=5,1 \mathrm{hr}$ on source, GOAL detector. 
groove shape for the wavelengths covered by the MCS. The cost of this improvement would be small compared to that of other developments.

As shown in Fig. 17, the dark current and readout noise in the MRS-L is comparable to or higher than the natural background noise. Regarding the dark current, the SOAP data were based on Spitzer Si:Sb array measurements collected by Van Cleve et al. (1995). Khalap \& Hogue (2012) reported several Si:Sb blocked impurity band (BIB) detector materials, which we have subsequently fabricated. We made a test device consisting of single-pixel structures from the detector material to measure dark currents. The measured dark current is lower than the GOAL. To set the GOAL dark current, a glow effect from the output FETs at the edge of the readout IC is added. However, this glow affects the dark level only near the detector edge. The echelle format of the MRS restricts its use to the inner part of the detector; therefore we can expect dark current somewhat smaller than the GOAL in MRS-L. The readout noise can be reduced by increasing the number of Fowler samples. We assumed that the readout noise is inversely proportional to the square root of $N_{F}$. In practice, this relationship depends on the frequency property of the detectors and should be characterized using the flight detectors.

\section{Summary}

We have designed MCS as a model instrument to investigate the performance of SPICA in the midinfrared region. SPICA is equipped with a $3-\mathrm{m}$ cooled telescope and four designed modules, two wide-field cameras with two independent $5 \times 5$ arcmin FOVs (WFC-S and WFC-L), a MRS that operates in the $12-38 \mu \mathrm{m}$ wavelength region (comprising MRS-S and MRS-L), and a HRS that operates in the $12-18 \mu \mathrm{m}$ region. Instrument sensitivities were estimated by state-of-the-art current technologies. The estimated performances are summarized in Table 15. A pointing correction system is required at the shorter half of the wavelength coverage. At wavelengths longer than $26 \mu \mathrm{m}$, improvement in the BPFs is highly desired, and the groove shape of the grating should be optimized to improve the throughputs.

\section{Acknowledgments}

This work is supported by grant MOST 100-2112M-001-001-MY3 (Y.O.).

\section{References}

Araki, K. [2005] Opt. Rev. 12, 219.

Bennett, J. M. \& Ashley, E. J. [1965] Appl. Opt. 4, 221.

Castel, D., Sein, E., Lopez, S., Nakagawa, T. \& Bougoin, M. [2012] "The $3.2 \mathrm{~m}$ all SiC telescope for SPICA" in Proc. SPIE, Vol. 8450, 84502P.

Fujishiro, N., Kataza, H., Wada, T. et al. [2012] "Free-form reflective optics for mid-infrared camera and spectrometer on board SPICA" in Proc. Int. Conf. Space Optics (ICSO 2012), p. 137.

Hawkins, G. \& Sherwood, R. [2008] Appl. Opt. 47, 25.

Khalap, V. \& Hogue, H. [2012] "Antimony doped Silicon Blocked Impurity Band (BIB) Arrays for low flux applications" in Proc. SPIE, Vol. 8512, 85120O.

Love, P. J., Beuville, E. J., Corrales, E. et al. [2006] "1024 $\times 1024$ Si:As IBC detector arrays for MID-IR astronomy" in Proc. SPIE, Vol. 6276, 62761Y.

Mahajan, V. N. [1983] J. Opt. Soc. Am. 73, 860.

Mainzer, A., Larsen, M., Stapelbroek, M. G. et al. [2008] "Characterization of flight detector arrays for the widefield infrared survey explorer" in Proc. SPIE, Vol. 7021, $70210 \mathrm{X}$.

Mitani, S., Kawakatsu, Y., Sakai, S.-I. et al. [2014] "Precision pointing control for SPICA: Risk mitigation phase study" in Proc. SPIE, Vol. 9143, 914347.

Nakagawa, T., Matsuhara, H. \& Kawakatsu, Y. [2012] "The next-generation infrared space telescope SPICA" in Proc. SPIE, Vol. 8442, 84420O.

Ootsubo, T., Onaka, T., Yamamura, I. et al. [2000] Adv. Space Res. 25, 2163.

Palner, C. H. \& Lebrun, H. [1972] Appl. Opt. 11, 907.

Reach, W. T., Morris, P., Boulanger, F. \& Okumura, K. [2003] Icarus 164, 384.

Ressler, M. E., Cho, H., Lee, R. A. M. et al. [2008] "Performance of the JWST/MIRI Si:As detectors" in Proc. SPIE, Vol. 7021, 70210 O.

Roelfsema, P., Giard, M., Najarro, F. et al. [2012] "The SAFARI Imaging Spectrometer for the SPICA space observatory" in Proc. SPIE, Vol. 8442, 84420R.

Sakon, I., Kataza, H., Onaka, T. et al. [2013a] "A design and trial production of the image slicer unit for the mid-infrared spectrograph" in Proc. SPIE, Vol. 8860, 88600Z.

Sakon, I., Onaka, T., Kataza, H. et al. [2013b] "Dust science with SPICA mid-infrared camera and spectrometers (MCS)" in Proc. The Life Cycle of Dust in the Universe: Observations, Theory and Laboratory Experiments (LCDU2013), Taipei, Taiwan, eds. Andersen, A., Baes, B., Gomez, H., Kemper, C., Watson, D., November 18-22.

Sakon, I., Onaka, T., Kataza, H. et al. [2014] "A trial production of the image slicer unit for next generation infrared instruments and the assembly of the evaluation system of 
the pseudo slit image quality" in Proc. SPIE, Vol. 9143, 91434U.

Schroeder, D. [2000] Astronomical Optics, Electronics \& Electrical (Academic Press, San Diego, CA).

Seeley, J. S., Hunneman, R. \& Whatley, A. [1981] Appl. Opt. 20, 31.

Swinyard, B. M., Rieke, G. H., Ressler, M. et al. [2004] "Sensitivity estimates for the mid-infrared instrument (MIRI) on the JWST" in Proc. SPIE, Vol. 5487, 785.
Van Cleve, J. E., Herter, T. L., Butturini, R. et al. [1995] "Evaluation of Si:As and $\mathrm{Si}: \mathrm{Sb}$ blocked impurity band detectors for SIRTF and WIRE" in Proc. SPIE, Vol. 2553, 502.

Wright, G. S., Rieke, G. H., Colina, L. et al. [2004] "The JWST MIRI instrument concept" in Proc. SPIE, Vol. 5487, 653. 